\title{
The structure and origin of confined Holmboe waves
}

\author{
Adrien Lefauve $^{1}$, Jamie L. Partridge ${ }^{1}$, Qi Zhou ${ }^{1, \dagger}$, S. B. Dalziel ${ }^{1}$, \\ C. P. Caulfield ${ }^{2,1}$, P. F. Linden ${ }^{1}$ \\ ${ }^{1}$ Department of Applied Mathematics and Theoretical Physics, Centre for Mathematical \\ Sciences, Wilberforce Road, Cambridge CB3 0WA, UK \\ ${ }^{2}$ BP Institute, University of Cambridge, Madingley Road, Cambridge CB3 0EZ, UK
}

(Received xx; revised xx; accepted xx)

Finite-amplitude manifestations of stratified shear flow instabilities and their spatiotemporal coherent structures are believed to play an important role in turbulent geophysical flows. Such shear flows commonly have layers separated by sharp density interfaces, and are therefore susceptible to the so-called Holmboe instability, and its finiteamplitude manifestation, the Holmboe wave. In this paper, we describe and elucidate the origin of an apparently previously unreported long-lived coherent structure in a laboratory stratified shear flow generated by exchange flow through an inclined square duct connecting two reservoirs filled with fluids at different densities. Using a novel measurement technique allowing for time-resolved, near-instantaneous measurements of the three-component velocity and density fields simultaneously over a three-dimensional volume, we describe the three-dimensional geometry and spatio-temporal dynamics of this structure. We identify it as a finite-amplitude, nonlinear, asymmetric confined Holmboe wave (CHW), and highlight the importance of its spanwise (lateral) confinement by the duct boundaries. We pay particular attention to the spanwise vorticity, which exhibits a travelling, near-periodic structure of sheared, distorted, prolate spheroids with a wide 'body' and a narrower 'head'. Using temporal linear stability analysis on the two-dimensional streamwise-averaged experimental flow, we solve for three-dimensional perturbations having two-dimensional, cross-sectionally confined eigenfunctions and a streamwise normal mode. We show that the dispersion relation and the three-dimensional spatial structure of the fastest growing confined Holmboe instability are in good agreement with those of the observed confined Holmboe wave. We also compare those results with a classical linear analysis of two-dimensional perturbations (i.e. with no spanwise dependence) on a one-dimensional base flow. We conclude that the lateral confinement is an important ingredient of the confined Holmboe instability, which gives rise to the CHW, with implications for many inherently confined geophysical flows such as in valleys, estuaries, straits or deep ocean trenches. Our results suggest that the $\mathrm{CHW}$ is an example of an experimentally observed, inherently nonlinear, robust, long-lived coherent structure which has developed from a linear instability. We conjecture that the CHW is a promising candidate for a class of exact coherent states underpinning the dynamics of more disordered, yet continually forced stratified shear flows.

\section{Key words:}

$\dagger$ Present address: Department of Civil Engineering, University of Calgary, 2500 University Drive NW, Calgary, Alberta T2N 1N4, Canada 


\section{Introduction}

The onset of instabilities triggering the transition to turbulence and consequent irreversible mixing in stratified shear flows, is a fundamental problem in fluid mechanics and was first recognised as such by Osborne Reynolds who observed instabilities on an interface between two immiscible fluids. In his classic paper, usually cited for his pipe flow experiments, Reynolds (1883) established a shear flow in a two-layer fluid by tilting a closed tube. He noted it was 'a very pretty experiment' which showed that 'there is a critical velocity ... at which direct motion becomes unstable' and that 'the instability ... did not depend on the magnitude of the disturbances'. The first application of linear stability theory to stratified shear flows was described by G. I. Taylor in his Adams Prize essay of 1915 entitled 'Turbulent motion in fluids'. Though Taylor published his stability predictions 'without waiting for experimental results' (Taylor 1931), the influential and beautiful 'tilting tank' experiments by Thorpe (1971) demonstrated that the development and turbulent break down of classical linear instabilities of stratified shear flows can be observed and measured in the laboratory. Since the pioneering work of Woods (1968), observational evidence has also accumulated that finite amplitude manifestations of stratified shear flow instabilities occur frequently in the oceans and atmosphere (see e.g. van Haren et al. 2014, Mahrt 2014). The mixing induced by the turbulence following these instabilities is thought to play a key role in diapycnal transport (see Ivey et al. 2008 for a review). Since turbulent mixing occurs on small scales relative to the current resolution of general circulation and climate models, the development of subgrid-scale parameterisations to model diapycnal mixing remains essential. However, there is still uncertainty concerning the causal relationship between bulk flow properties, spatio-temporally intermittent turbulence, and irreversible mixing.

A promising avenue of research to address this uncertainty is to analyse stratified turbulence in a dynamical systems framework, focusing on spatio-temporal structures rather than statistics. Within this approach, an interesting hypothesis is that the dynamics of turbulence can be described as an, in general complicated, trajectory in phase space that spends significant periods in the vicinity of a set of unstable nonlinear exact solutions of the Navier-Stokes equations called 'exact coherent states' (see e.g. Gibson et al. 2008). In certain circumstances, these exact coherent states can be traced back, generically through a sequence of bifurcations, to linear instabilities (Lucas et al. 2017). They can also provide useful insights into the properties of the turbulent attractor, for example by allowing for reduced-order-modelling of key quantities such as energy dissipation (Lucas \& Kerswell 2015) and irreversible mixing in stratified flows (Lucas \& Caulfield 2017). It is, however, proving extremely challenging to identify such exact coherent states in numerical simulations of flows prone to vigorous turbulence. This issue is particularly significant in stratified flows relevant to the ocean due to the large values of the Prandtl number $\operatorname{Pr} \equiv \nu / \kappa \approx 7$ and Schmidt number $S c \equiv \nu / \kappa_{s} \approx 700$, where $\nu$ is the kinematic viscosity, $\kappa$ is the thermal diffusivity and $\kappa_{s}$ is the salt diffusivity. The tendency of those flows to develop well-mixed layers separated by sharp density gradients (at least intermittently; see Salehipour et al. 2016), makes their direct numerical simulation beyond current computational capabilities. Therefore, laboratory experiments have an important role to play in guiding theoretical and numerical work towards the identification of dynamically important exact coherent states, under the hypothesis that they leave a distinctive signature in experimental turbulent flow, as observable, inherently nonlinear, yet relatively long-lived coherent structures.

In this paper, we describe an apparently previously unidentified coherent structure in a laboratory stratified shear flow. The stratified inclined duct experiment, described by 
Meyer \& Linden (2014), generates a two-layer stratified exchange flow in an inclined duct connecting two reservoirs containing fluids of different densities. As discussed in more detail by Meyer \& Linden (2014), variations in the density difference and/or inclination angle (a few degrees at most) lead to four qualitatively different observed flow states:

(i) laminar flow, characterised by a thin, flat density interface;

(ii) a largely coherent, but non-parallel flow state characterised by robust, propagating, quasi-periodic waves on the interface;

(iii) spatio-temporally intermittent turbulence with small-scale structures and mixing;

(iv) fully-developed turbulence with significant small-scale structures and a thick mixed interfacial density layer.

Here we focus on the coherent wave state (ii), which Meyer \& Linden (2014) described as the 'Holmboe regime', since the waves observed using a shadowgraph were very similar in appearance to the finite amplitude manifestation of this instability predicted by Holmboe (1962), observed experimentally by Thorpe (1968) in tilting tank experiments, and numerically simulated (in two-dimensional flows) first by Smyth et al. (1988). As we shall see, the coherent structure that we observe has close connections to the finite amplitude waves arising from the traditional (two-dimensional) Holmboe instability, but exhibits three-dimensionality and spanwise confinement within our experimental duct. Therefore, we refer to this structure as a 'confined Holmboe wave' (CHW). The aim of this paper is to characterise this structure, and to understand how it originates, in the specific sense of identifying and understanding the underlying physical mechanisms that lead to the observed behaviour.

We characterise the three-dimensional structure of the CHW using a novel technique allowing time-resolved near-instantaneous measurements of all three velocity components and density field over a three-dimensional volume. We demonstrate that many aspects of the observed CHW are consistent with the predictions of a linear normal mode stability analysis of the experimentally measured, quasi-steady background flow which develops within the flow. We call this instability the 'confined Holmboe instability' (CHI). Note that, in the rest of the paper, we aim to distinguish in general between

(i) an infinitesimally small perturbation that is linearly unstable, which we typically refer to as an instability;

(ii) a finite amplitude, nonlinear wave, which we typically refer to as a wave.

To address our above-mentioned aim of characterising and identifying the physical mechanisms at the origin of the $\mathrm{CHW}$ observed in the stratified inclined duct, the remainder of this paper is organised as follows. In $\S 2$ we review relevant previous work, particularly on the traditional, two-dimensional Holmboe instability, to contextualise and motivate our study. In $\S 3$ we describe the experiment and measurement technique, and present the experimental results to characterise the $\mathrm{CHW}$ in $\S 4$. In $\S 5$ we describe our linear stability analyses and compare their predictions (including the $\mathrm{CHI}$ ) with our experimental data in $\S 6$. Finally we present our conclusions, and suggest possible future research directions in $\S 7$.

\section{Context and motivation}

\subsection{Holmboe instabilities}

Holmboe (1962) first considered the stability of an idealised two-layer stratification embedded within a shear layer of finite depth, and demonstrated that, for sufficiently strong stratification, such inviscid flows are always linearly unstable over a finite band of wavenumbers. He considered a symmetric configuration, with the density interface 
located at the midpoint of the shear layer, so there are two normal modes (with equal and opposite phase speeds) with equal growth rates in this instability band of wavenumbers.

This instability, which we refer to as the 'traditional' Holmboe instability, can be interpreted physically as being due to a resonance between infinitesimal internal wave-like perturbations localised at the density interface and infinitesimal Rayleigh (or 'vorticity') wave-like perturbations localised at the edges of the shear layers (Caulfield 1994; Baines \& Mitsudera 1994). This interpretation is a particular example of the 'wave interaction theory' for the identification and classification of instabilities (see Carpenter et al. 2013 for a review). The symmetric problem considered by Holmboe (1962) has two such resonances simultaneously, leading to two instabilities: one localised in the upper half of the shear layer, the other in the lower half of the shear layer. However, this physical internal wave-vorticity wave interaction instability mechanism is generic and gives rise to instabilities sharing the same essential features as the traditional Holmboe instability in more general stratified shear flows with finite depth shear layers with embedded 'sharp' density interfaces. It is this generic class of instabilities that we, for simplicity, refer to as Holmboe instabilities (HIs).

Lawrence et al. (1991) generalised the original stability calculations of Holmboe to consider asymmetric flows where the sharp density interface and the midpoint of the shear layer are not coincident. This breaks the symmetry of the traditional stability problem, giving rise to two instabilities with different growth rates, phase speeds and wavenumber bands for a given stratification. For flows in domains that are not bounded vertically, the resonance between the infinitesimal internal wave-like perturbation localised at the density interface and the vorticity wave-like perturbation localised at the further edge of shear layer gives rise to the assumed dominant instability band, with larger maximum predicted growth rate, smaller magnitude phase speed and smaller wavenumbers. Haigh \& Lawrence (1999) further demonstrated that vertical confinement by sufficiently close top and bottom boundaries could switch the dominant branch of instability, consistent with the seminal work of Hazel (1972).

\subsection{Holmboe waves: laboratory observations and numerical simulations}

Finite amplitude manifestations of HIs, which we refer to as Holmboe waves (HWs), have been observed experimentally in a wide range of shear flows containing relatively sharp density interfaces (see e.g. Macagno \& Rouse 1961; Thorpe 1968; Browand \& Winant 1973; Maxworthy \& Browand 1975; Caulfield et al. 1995; Zhu \& Lawrence 2001; Hogg \& Ivey 2003; Tedford et al. 2009; Carpenter et al. 2010; Meyer \& Linden 2014). Generically, cusped waves are observed at the interface, propagating at a phase speed intermediate between the propagation speed of the density interface and the maximum speed of the sheared fluid, in qualitative agreement with linear stability theory.

Zhu \& Lawrence (2001) observed both symmetric (up-down) and asymmetric (only up or only down) HWs in a laboratory exchange flow. They compared their observations with two different normal mode stability calculations. One calculation used the piecewise-linear profiles originally considered by Holmboe (1962), while the other used smooth parallel velocity and density profiles, calculated as solutions to the Taylor-Goldstein equation by Nishida \& Yoshida (1990). They found only qualitative agreement with the predicted phase speeds and wavelengths, perhaps unsurprisingly due to the highly idealised nature of the theoretical calculations compared to the actual laboratory flows. Tedford et al. (2009) and Carpenter et al. (2010) conducted further experiments, and the latter made the first comparison with three-dimensional direct numerical simulations (DNS). They attributed the discrepancy between experiments and linear theory to wave stretching (increasing wavelength by the streamwise convective acceleration of the mean flow), and 
the discrepancy between DNS and linear theory to wave merging (increasing wavelength by vortex merging events). They also found that the wave amplitude was larger in the DNS than in the experiments, although the simulations did not correspond exactly to the experimental situation, in particular in the choice of the Schmidt number.

In the first two-dimensional DNS of HWs, Smyth et al. (1988) established that the counter-propagating trains of HWs above and below the (symmetrically located) density interface accelerate when the crests of the characteristic cusped waves are aligned vertically, and slow down when they are laterally far apart. As is apparent in their figure 7, this behaviour is associated with the relative location of the elliptical spanwise vortices that develop in front of the density crests, with the slowing occurring when the two vortices are close to being aligned vertically (corresponding to density crests being far apart). Indeed, for sufficiently weak stratification, these two vortices 'lock' and then roll up the interface into an array or 'train' of elliptical billows, i.e. the finite amplitude manifestation of the Rayleigh instability of a finite-depth shear layer (see Rayleigh 1879, and for further discussion of this phenomenon for 'marginal' HWs, Smyth \& Peltier 1991). Such structures are commonly referred to as 'Kelvin-Helmholtz billows', even though Kelvin (Thomson 1871) and Helmholtz (1878) only considered a discontinuous velocity profile, which does not exhibit the scale selection characteristic of billow trains.

The elliptical vortices of HWs develop close to the critical layers of the linear instability (i.e. where the predicted phase speed of the linear instability matches the flow speed). Due to the relatively strong baroclinic vorticity generation associated with the cusped waves, these vortices do not typically correspond to the maximum vorticity magnitude within the flow, although they typically induce the entrainment of 'wisps' of fluid from the crest of the propagating cusped waves.

Smyth \& Peltier (1991) used secondary linear Floquet stability analysis, (i.e. the linear stability analysis of non-parallel flow fields extracted from two-dimensional numerical simulations under the assumption that these flow fields were 'frozen' in time) in an attempt to understand how a two-dimensional saturated HW becomes three-dimensional, breaks down, and hence has the potential to undergo the transition to turbulence. Simulations at moderate Reynolds number suggested that HWs could be robust and identifiable over relatively long times (see for example Smyth \& Winters 2003, Carpenter et al. 2007 and Smyth et al. 2007). However, through simulations at significantly higher Reynolds number, Salehipour et al. (2016) demonstrated that finite amplitude symmetric HWs are prone to a wide range of secondary instabilities of both convective and sheardriven types, which destroy the coherence of the primary instability and trigger a relatively long period (at least in comparison to $\mathrm{KH}$ billow trains) of turbulent motions characterised by enhanced turbulent dissipation and irreversible mixing. Nevertheless, these behaviours at higher Reynolds numbers may well be inherently linked to the transient run-down nature of such simulations, with no forcing acting to re-energise the initial shear flow, and the initial profiles and computational domain geometry being chosen to be susceptible to specific (and typically monochromatic) linear instabilities.

\subsection{Limitations of previous research}

In this paper, we attempt to address some of the limitations of the previous studies of HWs, which we classify in three categories: steadiness; dissipation; and threedimensionality.

\subsubsection{Steadiness}

To the best of our knowledge, all numerical calculations have considered the time evolution of an initially unstable state, analogous to the time-dependent instabilities 
observed in 'tilting tank' experiments of Reynolds (1883) and Thorpe (1971). This setup is convenient for comparison with the predictions of linear stability theory in an initial value problem. However, many geophysically relevant flows - such as exchange flows in straits, estuaries (e.g. Geyer et al. 2010), coastal inlets (e.g. Farmer \& Armi 1999), deep ocean overflows (e.g. van Haren et al. 2014) and stratified flows in the atmospheric boundary layer (e.g. Mahrt 2014) - are sustained in time through slowly-varying forcing, such that a quasi-steady nonlinear state can exist. Such natural flows are not the product of carefully designed initial conditions chosen to trigger specific primary instabilities, which as noted above lead to inherently transient flow evolution and, in particular, preclude any possibility of finite-amplitude coherent structures emerging which are both robust and long-lived.

In contrast, the stratified inclined duct experiment sets up a statistically steady flow that is not the inevitable result of carefully designed initial conditions. HWs propagate throughout the duct and nonlinearly influence the mean flow that sustains them. This situation is more representative of environmental flows, yet there is no a priori rigorous argument justifying a connection between observed saturated nonlinear waves and linear instabilities predicted to grow from infinitesimal perturbations using the horizontallyaveraged mean flow as a notional, though not actually realised, base flow. In this paper we show that despite the undoubted challenge to this modelling approach, the comparison is, perhaps fortuitously, good.

\subsubsection{Dissipation}

The majority of the experiments and numerical calculations of which we are aware considered flows at relatively low Reynolds number of $O(10-100)$ (based on the definition (3.4) adopted later in this paper), with the recent exception of Salehipour et al. (2016). Moreover, the 'run-down' character means that a strictly restricted amount of energy, set at the beginning of the flow evolution, is available to dissipate, and that this dissipation is inherently limited in time. Indeed, the stratified turbulence literature (see e.g. the review of Ivey et al. 2008) increasingly highlights the importance of the 'buoyancy Reynolds number' $R e_{b}$, defined as

$$
R e_{b} \equiv \frac{\epsilon_{0}}{\nu N_{0}^{2}}
$$

Here, $\epsilon_{0}$ is a characteristic (volume-averaged) value of the rate of dissipation of total kinetic energy $\epsilon \equiv 2 \nu s_{i j} s_{i j}$ (where $s_{i j} \equiv\left(\partial_{x_{i}} u_{j}+\partial_{x_{j}} u_{i}\right) / 2$ is the symmetric strain rate tensor) and $N_{0}$ is a characteristic value of the buoyancy frequency $N \equiv \sqrt{-\left(g / \rho_{0}\right) \partial_{z} \rho}$. This nondimensional number, sometimes referred to as the 'activity parameter' or $G n$ (Portwood et al. 2016), is the (square) ratio of the frequencies associated with kinetic energy dissipation $\sqrt{\epsilon / \nu}$ and buoyancy $N$. An alternative and illuminating interpretation is to express $R e_{b}$ as a ratio of length scales: $R e_{b}=\left(L_{o} / L_{k}\right)^{4 / 3}$ where $L_{o}=\left(\epsilon / N^{3}\right)^{1 / 2}$ is the Ozmidov scale, the vertical scale below which turbulent eddies are not significantly affected by stratification, and $L_{k}=\left(\nu^{3} / \epsilon\right)^{1 / 4}$ is the Kolmogorov scale, below which viscous dissipation dominates. The ratio $R e_{b}$ therefore measures the range of scales that are not significantly affected either by stratification (suppressing vertical scales $\gtrsim L_{o}$ ) or by viscosity (damping scales $\lesssim 10 L_{k}$ ). Although caution needs to be exercised when comparing different studies and flow geometries where $R e_{b}$ may be defined in different ways, consensus is developing, not least motivated by the arguments of Gibson (1980) and Gibson (1999), that $R e_{b} \gtrsim 20-30$ is required for the flow to exhibit any of the key characteristics of stratified turbulence (see Portwood et al. 2016; Bartello \& Tobias 2013 for further discussion). 
In the stratified inclined duct experiment, we estimate the volume-averaged $R e_{b} \approx$ $R e \sin \theta$ (see (3.4) and (3.6)) and CHWs are found to exist for $R e_{b}$ up to $\approx 50$, controlled by increasing either the Reynolds number $R e$ or the tilt angle of the duct $\theta$ (see $\S 3.4 .1$ for more details). We are therefore able to generate waves that maintain relatively high dissipation for hundreds of advective times units, making this experiment a viable attempt to reproduce inertia-dominated flows of geophysical relevance in the laboratory.

\subsubsection{Three-dimensionality}

Temporal linear stability analyses of parallel shear flows with a background streamwise velocity $U(z) \hat{\mathbf{x}}$ focus on wave-like disturbances, i.e. normal modes, of the form

$$
\Psi^{\prime}\left(\mathbf{x}_{\perp}, z, t\right) \equiv \hat{\Psi}(z) \exp \left(i \mathbf{k} \cdot \mathbf{x}_{\perp}+\sigma t\right)
$$

where $\hat{\Psi}(z)$ is the one-dimensional complex eigenfunction of any perturbation variable; $z$ is the vertical coordinate; $\mathbf{x}_{\perp} \equiv x \hat{\mathbf{x}}+y \hat{\mathbf{y}}$ is the horizontal coordinate vector; $\mathbf{k} \equiv$ $|\mathbf{k}|(\cos \beta \hat{\mathbf{x}}+\sin \beta \hat{\mathbf{y}})$ is the real horizontal wave vector; and $\sigma$ is the complex growth rate. Conventionally, $\mathbf{k}$ is taken to be aligned with the background flow along $x$, i.e. $\beta=0$. In this paper, we refer to such an analysis as ' $2 \mathrm{P}-1 \mathrm{~B}$ ', for two-dimensional perturbations on a one-dimensional base flow and to the more general analysis with $\beta \neq 0$ as ' $2.5 \mathrm{P}$ 1B', for its intermediate character between two- and three-dimensional perturbations. The focus on $2 \mathrm{P}-1 \mathrm{~B}$ analyses can generically be justified by appealing to a corollary of 'Squire's theorem' (Squire 1933) in unstratified flows at finite Reynolds number, and the analogous 'Yih's theorem' (Yih 1955; Smyth et al. 1988) in (inviscid) stratified flow.

Both theorems rely on the observation that 'three-dimensional' normal modes aligned at some angle $\beta \neq 0$ to the background flow, having growth rate $\sigma$, wavenumber $|\mathbf{k}|$ in a flow with Reynolds number $R e$ and bulk Richardson number $R i$ are equivalent to 'twodimensional' normal modes having $\beta=0$ with lower growth rate $(\sigma \cos \beta)$ and larger wavenumber $(|\mathbf{k}| / \cos \beta)$ in an equivalent flow with lower Reynolds number $(\operatorname{Re} \cos \beta)$ and higher bulk Richardson number $\left(R i / \cos ^{2} \beta\right)$. This statement is summarised by the relation (see Smyth \& Peltier 1990):

$$
\sigma(|\mathbf{k}|, \beta, R e, R i)=\cos \beta \sigma\left(\frac{|\mathbf{k}|}{\cos \beta}, 0, R e \cos \beta, \frac{R i}{\cos ^{2} \beta}\right) .
$$

It is then natural to consider the locus of the two-dimensional modes $(\beta=0)$ with maximum growth rate $\sigma_{m}$ across all wavenumbers for given values of the external flow parameters $R e$ and $R i$. For an unstratified flow, if $\sigma_{m}(R e)$ decreases more slowly than $1 / R e$ (i.e. $\sigma_{m}(R e) \times R e$ increases with $R e$ ), then the most unstable two-dimensional modes have higher growth rates than any three-dimensional mode, a circumstance which is generic for shear flow instabilities. In particular, if instability occurs only above a critical Reynolds number (i.e. for $R e \geqslant R e_{c}$ ), this condition implies that the marginal instability mode must be two-dimensional (as any three-dimensional mode in a flow with $R e=R e_{c}$ experiences an equivalent flow with $R e<R e_{c}$, and hence is stable).

The equivalent statement for (inviscid) stratified flows is, if $\sigma_{m}(R i)$ increases more slowly than $\sqrt{R i}$ (i.e. $\sigma_{m}(R i) / \sqrt{R i}$ decreases with increasing $R i$ ), then the most unstable two-dimensional modes have higher growth rates than any three-dimensional mode. For stratified flows, the dominance of two-dimensional modes is not so clear-cut, as HIs (for example) only occur in stratified flows, and occur for all bulk Richardson numbers, thus making it at least plausible that $\sigma_{m}(R i)$ increases more rapidly than $\sqrt{R i}$. Indeed, in a stratified flow at finite Reynolds number, Smyth \& Peltier (1990) identified a small parameter range in $R e-R i$ space where $\sigma_{m}(R i)$ does increase more rapidly than $\sqrt{R i}$ 
and HIs at an angle $\beta \neq 0$ were predicted to be dominant, but it has proved difficult to test these predictions either experimentally or numerically.

It is important to recognise that such 'three-dimensional' modes considered by the $2.5 \mathrm{P}-1 \mathrm{~B}$ analysis (in this precise sense of travelling at angle $\beta \neq 0$ to the background flow) are strictly only well-defined in infinite domains with translational invariance in $x$ and $y$. As another example of a 'three-dimensional' stability analysis, Drazin (1974) considered the linear instability of an inviscid flow with two layers of different density, where the upper layer has a non-zero streamwise velocity which varies slowly in the spanwise direction. This flow is prone to a (truly) three-dimensional variant of the conventional Kelvin-Helmholtz instability, yet the background flow was still not confined or localised in any sense. Such flow instabilities, just like the flow instabilities 'propagating at an angle', are therefore unlikely to be observed experimentally. Furthermore, the modal structure (2.2) assumed for such perturbations inevitably imposes the same spanwise periodicity on the perturbation density and velocity components, thereby strongly restricting their possible spatial structure. As we discuss in more detail in this paper, perturbations with a more complex spatial structure are actually consistent with our experimental observations.

Indeed, experimental observations of HWs are typically compared to $\mathrm{HI}$ ' $2 \mathrm{P}-1 \mathrm{~B}$ ' predictions (often representing $U(z)$ by hyperbolic-tangent or error-function vertical profiles in unbounded domains). Analogously, three-dimensional numerical simulations typically consider periodic boundary conditions in both of the horizontal directions, avoiding potential issues with horizontal boundary conditions interacting with HWs. However, viscous effects and the presence of confining side walls in an experimental tank make any experimental mean flow inherently dependent on the spanwise $(y)$ and vertical $(z)$ coordinates and affect any waves which may develop by requiring the perturbations to decay to zero at these walls. Many of the geophysical flows mentioned in $\S 2.3 .1$ also exhibit two- or three-dimensional base flows and significant spatial confinement. It is therefore desirable to understand the effects of this inherent three-dimensionality and confinement and model them appropriately. Our experimental apparatus is designed to obtain data to achieve this.

\section{The experiment}

\subsection{The stratified inclined duct}

We consider the flow in a duct connecting two reservoirs containing aqueous salt solutions at densities $\rho_{0} \pm \Delta \rho / 2$ (figure 1). The seal, between the duct and the barrier separating the two reservoirs, is made of flexible rubber, allowing the duct to tilt at a relatively small angle $\theta$ from the horizontal. The duct has length $L=1350 \mathrm{~mm}$ and has a square cross-section of height and width $H=45 \mathrm{~mm}$ (aspect ratio $L / H=30$ ). At the start of the experiment, the duct is opened, initiating an exchange flow (with zero net volume flux) between the reservoirs.

This flow is a two-layer stratified shear flow, forced by gravity in two distinct ways. The first contribution is a hydrostatic pressure gradient in each layer, resulting from the end of each duct sitting in reservoirs containing fluids of different densities, and is sufficient to drive an exchange flow at $\theta=0^{\circ}$. The second contribution depends on the tilt angle: the resulting non-zero projection of gravity along the duct provides additional buoyancy forces. A positive angle $\theta>0^{\circ}$ (defined here by the duct being raised in the denser reservoir) reinforces the exchange flow by accelerating the light layer up (to the left) and the heavy layer down (to the right). Each individual reservoir measures $1 \times 0.2 \times 0.5 \mathrm{~m}$ and 

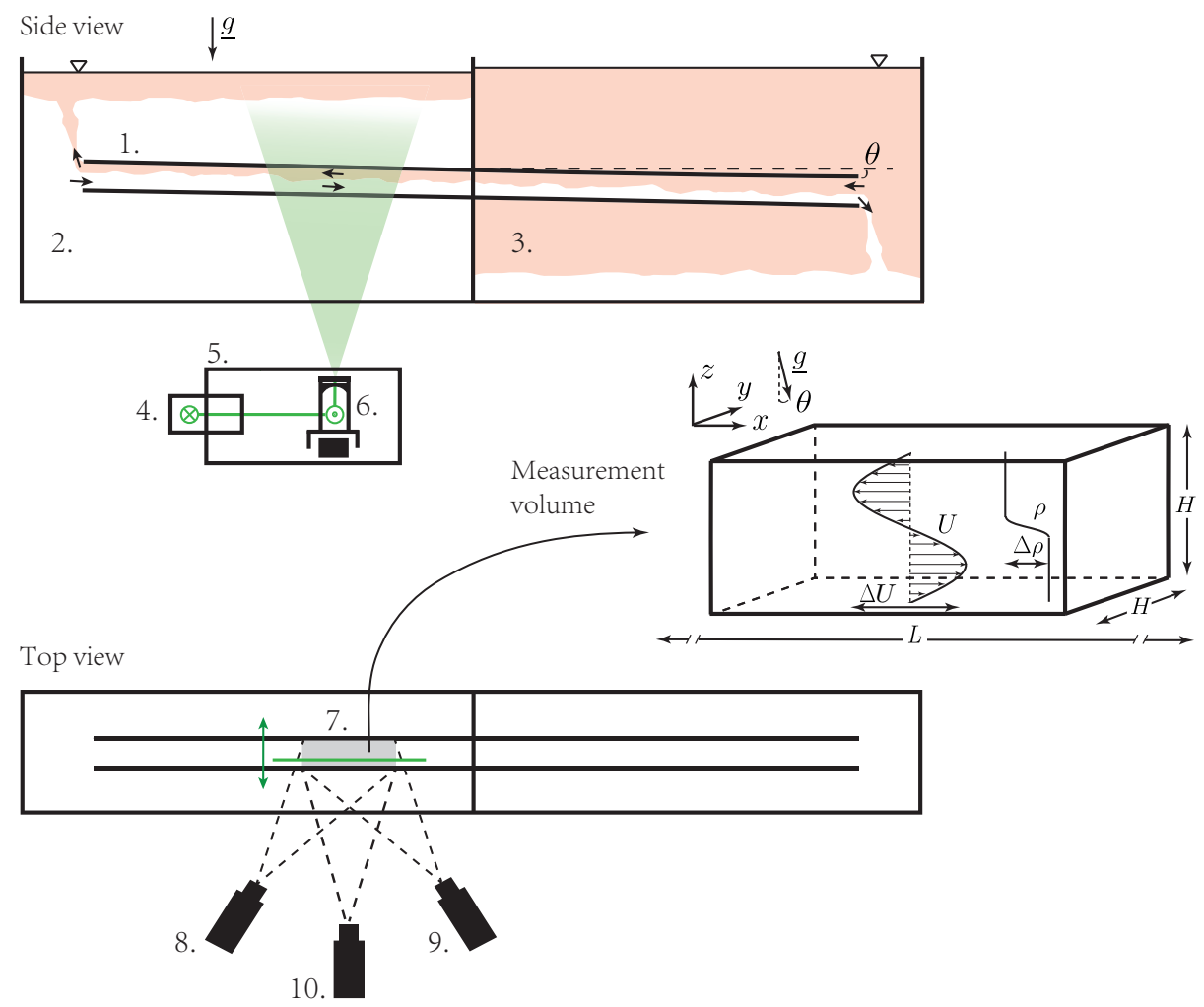

FiguRE 1. Experimental flow geometry: An inclined duct (1) connects a reservoir of dense fluid (2) and a reservoir of light fluid (3). All fluid is seeded with particles for sPIV, and fluid initially in (3) is dyed for PLIF. The laser beam emitted from (4) is directed to the pair of oscillating mirrors in (5), before entering the light sheet producing optics mounted on the linear traverse (6). The scanning light sheet sweeps a measurement volume (7) whose successive planes are imaged for sPIV by cameras (8) and (9) and for PLIF by camera (10).

holds approximately 100 litres, allowing us to maintain a statistically-steady exchange flow for several minutes. This steady flow continues until the controls at the ends of the duct (see below) are flooded by the accumulation of fluid of a different density coming from the other reservoir.

As outlined in $\S 1$, Meyer \& Linden (2014) described and mapped four qualitatively different flow states obtained by varying the main parameters $\Delta \rho / \rho_{0}$ and $\theta$, using shadowgraph observations and a larger square duct with length $L=3000 \mathrm{~mm}$ and height $H=100 \mathrm{~mm}$. At low $\Delta \rho / \rho_{0}$ and $\theta$, the flow is laminar with an undistorted interface. At larger values of either or both parameters, persistent coherent HWs distort the interface. Higher values of the forcing give rise to spatio-temporally intermittent turbulence at the interface, and eventually, fully developed turbulence with a thick mixing layer. Their measurements of layer-averaged velocities through mass flux balances support the hypothesis that the flow is hydraulically controlled at both ends of the duct for $\theta \gtrsim 1^{\circ}$. The flow is subcritical (with respect to long, small amplitude, interfacial disturbances) inside the duct, and undergoes two transitions at each end: a smooth one to a supercritical state shortly after exiting the duct, and an abrupt one further downstream (in the form of a jump) from supercritical to subcritical, to match the reservoir conditions. These hydraulic controls ensure that the exchange flow taking place through the duct is 
maximal, i.e. that its layer-averaged velocities obey the Froude number condition (3.1) (see Armi 1986).

The practical consequence of this hydraulic control is that positive angles cannot increase the exchange flow rate, or mean flow velocities, beyond a certain value. The additional kinetic energy input provided by gravity through a positive tilt angle must be dissipated by the flow within the duct, either by increased wave activity or turbulence. We are therefore able to control the dissipation rate by the tilt angle, and to maintain target dissipation rates in a statistically steady sense for extended periods of times. The dissipation achieved is expressed non-dimensionally as a buoyancy Reynolds number in $\S 3.2$, and depends on two free experimental parameters: the Reynolds number (when appropriately defined as below) and the tilt angle.

The inclined duct experiment is thus ideally suited to study HWs for the following reasons:

(i) It naturally sets up a flow with large shear layer thickness/density interface thickness ratio. The low diffusivity of salt compared to momentum (or high Schmidt number $S c \approx 700$, as defined in $\S 1$ ), and the continuous supply of unmixed fluid from both reservoirs in the open duct mean that the interface thickness ratio resulting from interfacial diffusion of vorticity compared to interfacial diffusion of density is of the order of $\sqrt{S c}=O(10)$.

(ii) It naturally sets up a flow with large bulk Richardson number. The hydraulic control sets an upper bound for the speeds achieved inside the duct and, as we shall see in $\S 3.2$ and from (3.5), effectively sets the overall or bulk Richardson number to an $O(0.1-1)$ constant, regardless of the forcing parameters.

(iii) Both symmetric and asymmetric $H W$ s can be observed. Experiments at low angles $\theta \lesssim 2^{\circ}$ typically exhibit flows where the density interface is (close to) the centre of the shear layer and so is prone to symmetric HWs (i.e. two waves propagating in opposite directions with similar amplitude). In contrast, experiments at higher angles $\theta \gtrsim 2^{\circ}$ exhibit an offset between the midpoint of the shear layer and the density interface sufficient to give rise to asymmetric (i.e. one-sided) HWs, where the instability with smaller growth rate is apparently being sufficiently suppressed such that it does not reach an observable 'wave' with finite amplitude. The mechanism leading to the offset of the interface is thought to be related to the complex pressure adjustment necessary to match the different hydrostatic pressures in each reservoirs when the duct is tilted. We will use this empirical knowledge to our advantage in this paper, deliberately selecting parameters for which asymmetric HWs are observed.

\subsection{Notation and nondimensional parameters}

The notation we use in the paper is shown in the measurement volume insert in figure 1. The streamwise $x$ axis is aligned along the duct and the spanwise $y$ axis across the duct, making the $z$ axis tilted at an angle $\theta$ from the vertical (resulting in a non-zero streamwise projection of gravity). All coordinates are centred in the middle of the duct, such that $-L / 2 \leqslant x \leqslant L / 2$ and $-H / 2 \leqslant y, z \leqslant H / 2$. The velocity vector field has components $\mathbf{u}(x, y, z, t)=(u, v, w)$ along $x, y, z$, respectively, and we denote the density field by $\rho(x, y, z, t)$.

Only a limited number of parameters are believed to play important roles in this experiment. The geometrical parameters are $L, H, \theta$ and the dynamical parameters are the acceleration due to gravity $g$, the non-dimensional density difference $\Delta \rho / \rho_{0}$, the kinematic viscosity of water $\nu$ and the molecular diffusivity of salt $\kappa_{s}$. The relative density difference $(<0.1 \%$ in the experiment reported here) is sufficiently small so that the Boussinesq approximation requiring that $\Delta \rho / \rho_{0} \ll 1$ is valid and density differences 
only play a dynamically relevant role through the reduced gravity $g^{\prime} \equiv g \Delta \rho / \rho_{0}$. Since the six free parameters $L, H, \theta, g^{\prime}, \nu, \kappa_{s}$ have two independent dimensions (of length and time), it is possible to construct four independent nondimensional parameters, as we do below.

It is important to note at this point that the experiment does not have any imposed velocity scale. However, to a good approximation, the hydraulic controls at the ends of the duct require that the composite Froude number

$$
G^{2} \equiv F_{1}^{2}+F_{2}^{2}=1
$$

where $F_{i}^{2} \equiv\left\langle u_{i}^{2}\right\rangle_{y, z} /\left(g^{\prime} h_{i}\right)$ is the Froude number of layer $i$ and $\langle\cdot\rangle_{y, z}$ denotes spanwise and vertical averaging over the depth $h_{i}$ of each layer. The symmetry of this Boussinesq exchange flow implies that both layers have equal depth and Froude number in the central section of the duct

$$
F^{2} \equiv F_{1}^{2}(x=0)=F_{2}^{2}(x=0)
$$

such that for each layer

$$
F=\frac{\langle u\rangle_{y, z}}{\sqrt{g^{\prime}(H / 2)}}= \pm \frac{1}{\sqrt{2}}, \text { or }\langle u\rangle_{y, z}= \pm \frac{\sqrt{g^{\prime} H}}{2} .
$$

Due to the moderate values of $R e$, the mean velocity profile is affected by viscosity and we find that the peak velocities in each layer are approximately twice the layeraveraged values, i.e. $\max |u| \approx \sqrt{g^{\prime} H}$. We choose to nondimensionalise velocities by this characteristic peak value, or half the total (peak-to-peak) velocity jump $\Delta U / 2 \equiv \sqrt{g^{\prime} H}$. We define $(\tilde{u}, \tilde{v}, \tilde{w}) \equiv(u, v, w) /(\Delta U / 2)$ such that $-1 \lesssim \tilde{u} \lesssim 1$ (note that in general $|\tilde{v}|,|\tilde{w}| \ll|\tilde{u}|$; see $\S 6.1 .2)$. For consistency, we choose $H / 2$ as the length scale, such that $-1 \leqslant \tilde{y}, \tilde{z} \leqslant 1$, and $-L / H \leqslant \tilde{x} \leqslant L / H$. Consequently, the natural time scale for nondimensionalisation is the advective time $H / \Delta U=H /\left(2 \sqrt{g^{\prime} H}\right)$. The resulting nondimensional time units will be referred to as advective time units (ATU). The relevant Boussinesq density field is $\tilde{\rho} \equiv\left(\rho-\rho_{0}\right) /(\Delta \rho / 2)$, where we use $\Delta \rho / 2$ to nondimensionalise density such that $-1 \leqslant \tilde{\rho} \leqslant 1$.

Using the previously defined scales, it is natural to construct the following Reynolds number

$$
R e \equiv \frac{\frac{\Delta U}{2} \frac{H}{2}}{\nu}=\frac{\sqrt{g^{\prime} H} H}{2 \nu}
$$

which, for a given duct geometry and fluid, is a function of the driving density difference $\Delta \rho$ alone.

The Froude condition (3.1) artificially adds another dimensional parameter, the velocity scale, to our previous set of six parameters. It will prove useful in the rest of the paper (e.g. for the governing equations (5.1)) to define the overall Richardson number of the flow, expressed as the nondimensional product of the density, length and inverse square velocity scales

$$
R i \equiv \frac{\frac{g}{\rho_{0}} \frac{\Delta \rho}{2} \frac{H}{2}}{\left(\frac{\Delta U}{2}\right)^{2}}=\frac{1}{4}
$$

by definition of $\Delta U$. Note that the value of $R i$ is set and not a free parameter; it is simply an equivalent formulation of the Froude condition (3.1): $R i=1 /\left(8 F^{2}\right)=1 /\left(4 G^{2}\right)$ and $G^{2}=1$. We choose the Schmidt number $S c$ (defined in $\S 1$ ), $L / H$ and $\theta$ as the last parameters. In summary, we have a total of four free independent nondimensional parameters: $S c, L / H, \theta, R e$, and one imposed parameter $R i$. For the apparatus considered, 
we have $S c=700, L / H=30, R i=1 / 4$, and we have the freedom to vary $\theta$ and $R e$ (through varying $\Delta \rho$ ).

A characteristic buoyancy Reynolds number $R e_{b}$ (see $\S 1$ and (2.1)) can be estimated for $\theta>0^{\circ}$, assuming complete dissipation of the instantaneous power gained by the fluid moving at a layer-averaged velocity $\Delta U / 4$ in the streamwise gravity field $g^{\prime} \sin \theta$, i.e. the characteristic dissipation scale is $\epsilon_{0} \approx g^{\prime}(\Delta U / 4) \sin \theta$. Using the characteristic buoyancy frequency $N_{0}^{2}=g \Delta \rho /\left(2 \rho_{0}\right) /(H / 2)=g^{\prime} / H$, we find that

$$
R e_{b} \approx \frac{g^{\prime} \frac{\Delta U}{4} \sin \theta}{\nu \frac{g^{\prime}}{H}}=\frac{\Delta U H}{4 \nu} \sin \theta=\operatorname{Re} \sin \theta .
$$

Note that this is a volume-averaged estimate that includes the dissipation due to viscous stresses on the duct walls, and that the actual dissipation can be highly heterogeneous in space. Therefore, it is necessary to be cautious when comparing the specific numerical values of $R e_{b}$ reported here with other studies where other definitions have been used. For positive angles $\theta>0^{\circ}$, the three main qualitative bifurcations in flow regimes have been found to scale with $R e \sin \theta$ over a wide range of $R e$ and $\theta$. Based on hundreds of shadowgraph observations, HWs are observed for $20 \lesssim R e_{b} \lesssim 50$, intermittent turbulence is observed for $50 \lesssim R e_{b} \lesssim 100$ and steady turbulence for $R e_{b} \gtrsim 100$ (Lefauve 2018).

Henceforth, we drop the tildes and, unless explicitly stated otherwise, use nondimensional variables throughout.

\subsection{Three-dimensional volumetric sPIV/PLIF measurements}

We obtained time-resolved, three-dimensional, volumetric measurements of the threecomponents of velocity using a novel method that combines successive two-dimensional planar stereo particle image velocimetry (sPIV) measurements to construct a volume. The flow was illuminated by a thin, pulsed, vertical laser sheet, which was rapidly scanned back and forth in the spanwise $y$ direction (figure 1), sweeping the volume of interest. The fundamental technical challenge with this approach is to obtain pairs of images, separated by a small time interval appropriate for PIV, at the same spanwise locations without relying on the overlap of successive laser sheets achieved either from the continuous traverse of an excessively thick laser sheet (which would compromise the spanwise resolution) or from a very slow scanning speed (which would compromise the near-instantaneous character of the measurements). The novelty of the system employed here is the conversion of the continuous motion of the traverse into a discontinuous motion of the light sheet. The laser beam was directed onto a pair of oscillating mirrors controlled by galvanometers (for accurate and fast positioning) that deflect its position before entering the optics mounted on the fast-moving linear traverse. This allowed us to control the successive positions of the pulsed laser sheet independently of the traverse, and thus obtain pairs of images at the same spanwise location while continuously scanning the thin laser sheet across the measurement volume. A detailed description of the scanning system and other aspects of the sPIV/PLIF measurements can be found in Partridge et al. (2018)).

The disadvantage of this approach is that the measurements are not instantaneous as successive planes have a time lag. However, this time lag can be made very small by increasing the scanning speed while maintaining the co-location of laser sheet pairs. As discussed in more detail in Partridge et al. (2018), this method has three main advantages over existing methods for obtaining three-dimensional experimental diagnostics of a stratified flow across a volume: it is less sensitive to unavoidable residual refractive 
index variations; it yields a higher spatial resolution; and crucially, it allows simultaneous measurements of the density field.

Simultaneous measurements of the density field were achieved by combining successive planar laser induced fluorescence (PLIF) measurements in the same fashion. PLIF requires an additional camera imaging the concentration of a fluorescent dye marking one of the fluids (in our case, the less dense solution, initially in region (3) of figure 1). The dye emits light at a slightly longer wavelength than the wavelength of the laser light which the dye absorbs. Narrow bandpass filters in front of the PIV cameras and a suitable longpass filter for the PLIF camera allow separation of both signals.

\subsection{Experimental details}

\subsubsection{Flow parameters}

As discussed in $\S 3.2$, HWs are observed for a wide range of parameters $(\theta, R e)$. For $\theta=0^{\circ}$, the HWs are typically symmetric and are observed for $2000 \lesssim R e \lesssim 4500$. For $\theta=5^{\circ}$ the HWs are typically asymmetric and observed for $300 \lesssim R e \lesssim 600$.

In order to gain insight into the three-dimensional structure of $\mathrm{CHWs}$, analysing the propagation of an asymmetric Holmboe wave train, propagating only in one direction proves more straightforward. Moreover, the current limitation of our system in terms of scanning speed and the maximum displacement that can be achieved by the oscillating mirror means that a forward or backward scan cannot be achieved in less than approximately one second. At the current values of $H$ and $\nu$, a one-second scanning duration corresponds to $\Delta U / H=4 \nu R e / H^{2}=R e / 482$ ATU, making the measurement of flows with, for example, $R e=O(500)$ in $O(1$ ATU $)$ more reasonably assumed to be near to instantaneous than those with $R e=O(5000)$ in $O(10$ ATU $)$. For these reasons, the principal experiment discussed in this paper was carried out at $\theta=5^{\circ}, R e=440$, corresponding to $R e_{b}=38$.

Four additional experiments were carried out with the same parameters except for slightly different $R e \in[400,490]$ and initial transients resulting from different experimental initialisation procedures. The data from these additional experiments confirm that the conclusions drawn from the principal experiment presented in this paper are robust. For the sake of brevity, and also because the data from these additional experiments are of inferior quality by comparison to the data from the principal experiment, we do not discuss these experiments further.

\subsubsection{Fluids}

The success of both the sPIV and PLIF measurements relies on both fluids having very similar refractive indices, such that both particles and dye concentration can be accurately imaged without aberrations. Two salt solutions were used for that purpose: sodium chloride $(\mathrm{NaCl})$ for the light layer and sodium nitrate $\left(\mathrm{NaNO}_{3}\right)$ for the heavy layer. Using a handheld refractometer (Reichert Technologies Goldberg) it was possible to obtain the desired density difference $\Delta \rho$ while matching refractive indices within a relative error of $\Delta n / n \approx 10^{-4}$, small enough to obtain sharp and accurate particle images. The difference in diffusivity of these solutions is negligible, and does not introduce any additional dynamics, as discussed in Olsthoorn \& Dalziel (2017).

Densities were measured at the temperature of $20{ }^{\circ} \mathrm{C}$ (at which the experiment was carried out) using a density meter (Anton Paar DMA 5000). Here $\rho_{0}-\Delta \rho / 2=1.003242$ $\mathrm{g} \mathrm{cm}^{-3}$ and $\rho_{0}+\Delta \rho / 2=1.004190 \mathrm{~g} \mathrm{~cm}^{-3}$, giving $\Delta \rho / \rho_{0}=9.44 \times 10^{-4}$ and $R e=440$ using an average viscosity $\nu=1.05 \times 10^{-6} \mathrm{~m}^{2} \mathrm{~s}^{-1}$. The angle of the duct was set to $\theta=5.0^{\circ}$ using a digital inclinometer (Digi-Pas DWL-280Pro). 
Both fluids were seeded with polyamide particles of diameter $d_{p}=50 \mu \mathrm{m}$ and density $\rho_{p}=1.03 \mathrm{~g} \mathrm{~cm}^{-3}$ for PIV. Their small size and near-neutral density ensured that they accurately followed the flow, as evidenced by the small ratio of settling to mean flow velocities, or Stokes number $S k=\left(\rho_{p}-\rho_{0}\right) g d_{p}^{2} /\left(9 \rho_{0} \nu \Delta U\right)=1.7 \times 10^{-3}$. The less dense layer was dyed with rhodamine $6 \mathrm{G}$ at $1.5 \times 10^{-8} \mathrm{~g} \mathrm{~cm}^{-3}$ for PLIF, a low enough concentration to produce no visible attenuation of the laser sheet over the height of the duct, nor to affect the density of the fluid.

\subsubsection{Optics}

The light sheet was produced by a dual-cavity Litron Nano L 100 Nd:YAG laser, providing $50 \mathrm{~mJ}$ per pulse of duration $O(10 \mathrm{~ns})$ for each head, here used at a frequency of $50 \mathrm{~Hz}$. The thickness of the laser sheet varied between $\approx 2 \mathrm{~mm}$ at the centre and $\approx$ $1 \mathrm{~mm}$ at the edges of the measurement volume.

Experiments were recorded using three 8 MPixel Teledyne Dalsa Falcon2 cameras, each connected to a workstation and triggered by a timing system for accurate laser pulse camera trigger synchronisation, handled via the DigiFlow software. The same timing system controlled the galvo-mirrors and traverse responsible for scanning the laser sheet. The two PIV cameras were fitted with Micro Nikkor $60 \mathrm{~mm} \mathrm{f/2.8D} \mathrm{lenses} \mathrm{at} \mathrm{aperture}$ $\mathrm{f} / 8$ and were positioned at a distance of $0.6 \mathrm{~m}$ from the measurement volume. As those cameras must image the flow at an angle (figure 1), Scheimpflug adaptors were fitted in order to keep focus on oblique planes. The PLIF camera was fitted with a Nikkor $50 \mathrm{~mm}$ $\mathrm{f} / 1.2 \mathrm{D}$ at aperture $\mathrm{f} / 1.2$ and positioned at a distance of $1 \mathrm{~m}$ from the measurement volume. With these parameters, all cameras maintained good focus on all the planes scanned. As explained above, the cameras were equipped with filters to separate the signals from either the particles or the dye.

To ensure that particles in the reservoir did not interfere with PLIF measurements by blocking light coming from the duct, a box filled with pure water was inserted between the duct outer wall and the reservoir inner wall (not shown in figure 1) to allow an unobstructed view of the test section. This box, as well as the duct and reservoirs, is made of smooth, transparent Perspex (acrylic) sheets with good optical clarity.

\subsubsection{Scanning parameters and coordinates}

The scanning system was set up so that a measurement volume was spanned by 30 parallel planes to avoid redundancy of data by overlapping light sheets (average spanwise separation $=45 / 30=1.5 \mathrm{~mm}$, which is the average light sheet thickness). Therefore, to construct each volume, a forward scan or a backward scan captured 60 frames (noting that two frames are needed for each velocity slice). At a camera speed, or capture rate, of $50 \mathrm{fps}$ (for adequate particle displacement between frames) each forward or backward scan therefore took $1.2 \mathrm{~s}$ to complete, or 1.1 advective time units (ATU). This proved to be fast enough to 'freeze' adequately the structure of waves travelling at the (relatively small) phase speed of the order of $5-10 \%$ of the maximum advective speed, as our data will show.

A total of 309 volumes were captured, spanning 370 s or 335 ATU and representing $150 \mathrm{~GB}$ of raw data. The recording was started at an arbitrary time origin $t=0$ once the flow was established, a few seconds after the gravity current following the opening of one end of the duct had exited the other open end of the duct. The measurement volume spanned $248 \mathrm{~mm}$ in the streamwise direction and the full cross-section $45 \times 45 \mathrm{~mm}$, or $11 \times 2 \times 2$ in non-dimensional units. The corresponding coordinates of our measurement volume are $(x, y, z, t) \in[-17.5,-6.5] \times[-1,1] \times[-1,1] \times[0,335]$, where $(x, y, z)=(0,0,0)$ is the centre of the duct. 


\subsubsection{Data processing and resolution}

In order for multi-plane measurements in pixel coordinates from three cameras to be mapped into a three-dimensional volume with real world coordinates, a careful calibration technique was employed. It relied on using a two-plane calibration target, each plane containing approximately 100 white circles on a black background at known positions, inserted inside the duct prior to the experiment. The process of capturing still images of the target was repeated by shifting the target in the spanwise direction to three different $(x, z)$ planes inside the duct to obtain a set of approximately 600 points used to fit a least-square polynomial mapping between pixel and $(x, y, z)$ world coordinates. Given the importance of an accurate mapping for sPIV reconstruction, and since the light sheet is not infinitely thin, a further step of coordinate mapping refinement was used, as discussed in Partridge et al. (2018).

Initial, two-dimensional, sPIV processing for each camera was carried out using the DigiFlow software using a multi-pass adaptive algorithm that includes the distortion of interrogation windows. Here we used a nominal window size of $31 \times 31$ pixels and a spacing of 8 pixels. The pixel-based velocity fields from each camera were then combined using the coordinate system, again in DigiFlow, to produce the three-dimensional velocity field for each plane. By aggregating fields from across a scan, a final resolution of $440 \times 30 \times 70 \times 309$ is achieved in $x, y, z, t$, corresponding to a vector spacing of $0.025 \times 0.067 \times 0.028 \times 1.08$ (or $0.55 \mathrm{~mm} \times 1.5 \mathrm{~mm} \times 0.64 \mathrm{~mm} \times 1.2 \mathrm{~s}$ ). For comparison, the Kolmogorov scale (see $\S 2.3 .2$ ) in this flow is measured as $L_{k}=0.032$ (or $0.72 \mathrm{~mm}$ ), hence our streamwise and vertical resolutions are better than $L_{k}$, while our spanwise resolution is around $2 L_{k}$. Prior to computing vorticity using second-order finite differences, the velocity field is filtered in each $(x, z)$ plane using an isotropic two-dimensional Gaussian filter with modest standard deviation of 1 vector spacing. Vorticity data are then shown raw, except for the threedimensional isosurfaces, which were smoothed by an isotropic three-dimensional Gaussian filter with standard deviation of 1 vector spacing.

PLIF post-processing was used to deduce the density field from the imaged dye fluorescence. A careful calibration step was required, using full volume scans of images in which the duct is empty and then full of dye. The minimum (background) and maximum light intensity were used to rescale the dye images, in order to account for non-uniformity and divergence of the light sheet. Particles sitting in the tank below the duct interfered with the incoming light sheet and generated spatio-temporally dependent tilted rays, fanned out by the cylindrical sheet-producing optics. These artefacts were removed on all frames by first projecting the processed images into ray coordinates (making the rays vertical), then normalising by a reference image produced by vertically averaging a section in the upper layer (which should always be of uniform density in this twolayer flow) before finally being projected back to world coordinates. Only one of the two PLIF planes obtained for each sPIV plane was used, as the second one was found to contain little additional information. Due to its higher $(x, z)$ resolution compared with the velocity field (around six times higher in both $x$ and $z$ ), the density field was first filtered in each plane using a median filter of size $3 \times 3$ pixels, before being interpolated onto the grid of the sPIV data.

\section{Experimental results}

\subsection{Instantaneous snapshots}

Figure 2 shows four snapshots of the flow (in nondimensional units), in the vertical mid-plane of the duct $y=0$. The spanwise component of vorticity $\omega_{y}=\partial_{z} u-\partial_{x} w$ (left 

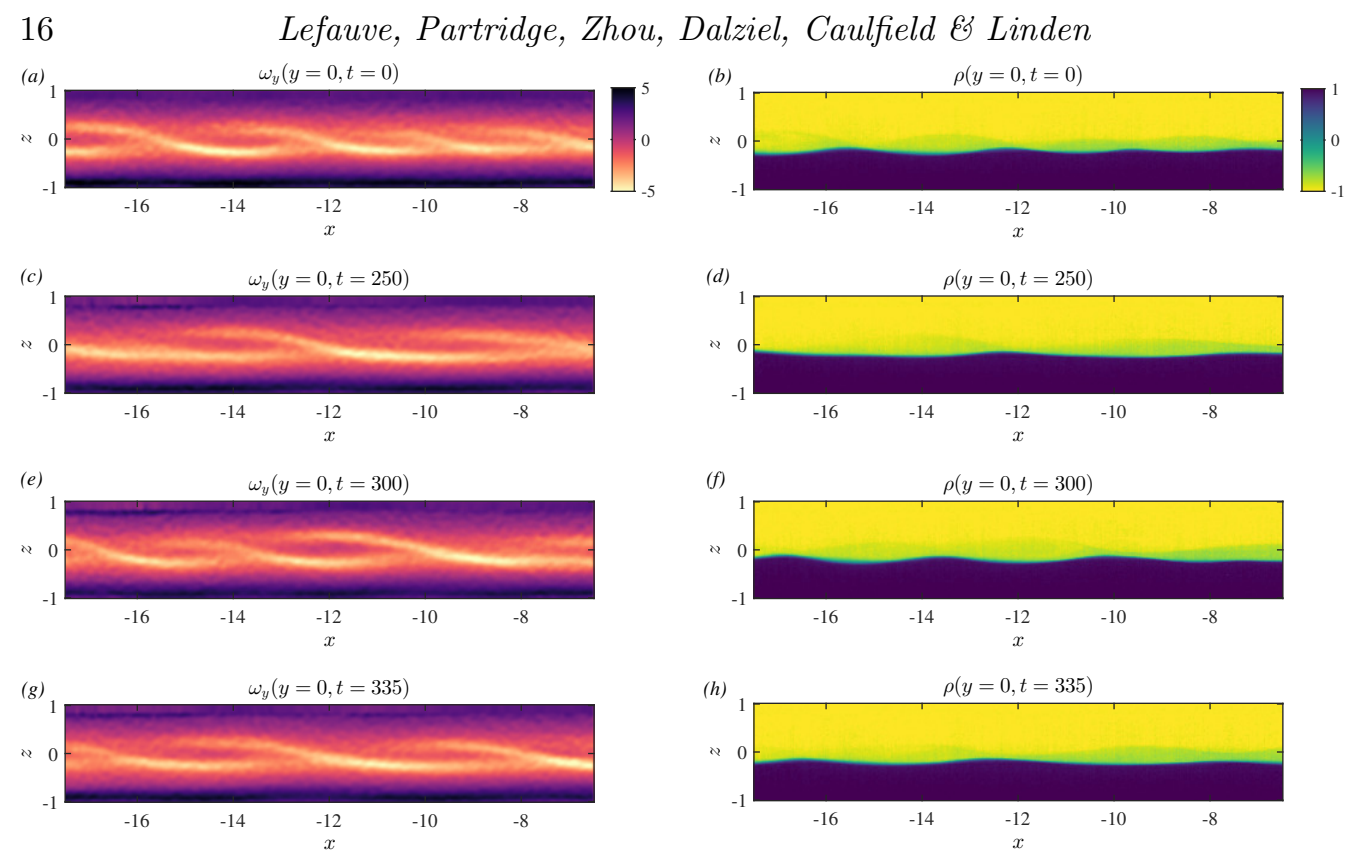

FiguRE 2. Snapshots of spanwise vorticity $\omega_{y}$ (left column) and density $\rho$ (right column) in the mid-plane $y=0$ at $t=0$ (a-b), $t=250(\mathrm{c}-\mathrm{d}), t=300$ (e-f) and $t=335(\mathrm{~g}-\mathrm{h})$. Axes are to scale. Throughout the paper, all data are shown in nondimensional units.

panels) is shown together with the density field $\rho$ (right panels) at four different times $t=0,250,300,335$ (our choice of times is motivated by the analysis in $\S 4.3$ ). A movie showing the full dynamics is available as supplementary movie 1. Both fields exhibit a quasi-periodic wave pattern corrugating the interface by upward-pointing cusps that propagate leftwards (with negative phase speed), as will be shown in $\S 4.2$. The vorticity field shows concentration of negative vorticity that spans the interface downward to the right in tilde-shaped bands. The wavelength of these patterns shows some temporal variation, but both fields are coupled in the sense that their wavelength changes together. It is this wave that we refer to as a confined Holmboe wave (CHW).

A small amount of mixed fluid is present above the sharp interface, consistent with thin wisp-like ejections characteristic of asymmetric HWs, as discussed by Carpenter et al. (2007) (see their figure 19). No wisp ejection was detected within the measurement volume; mixed fluid was either advected from other regions inside the duct where ejection took place and/or was a residual from the start of the experiment that had not been flushed out due to small velocities at the density interface.

To gain insight into the three-dimensional structure of the vorticity field, figure 3 shows, for the same times, the isosurface $\omega_{y}=-\omega_{\max } / 2=-2.5$, where $\omega_{\max }=5.0$ is the maximum value of $\left|\omega_{y}\right|$ found in the domain. This value represents a good intermediate value that shows the structure of interest. (Values $\omega_{y} \gtrsim-2$ mostly show the $x$-independent mean shear while values $\omega_{y} \lesssim-3$ show only a small part of the wave structure.) To make observation easier, the $z$ axis is stretched by a factor of three, so it is important to remember that the actual structure is closer to horizontal than displayed.

The vorticity structure is confined both in the spanwise $(y)$ and vertical $(z)$ directions and takes the form of inclined, distorted, prolate spheroids with a wide (in $y$ ) 'body' and a progressively (in the negative $x$ direction) narrower 'head' (as indicated by the arrows in figure $3(\mathrm{a})$ ). A chevron shape at the posterior side of the body is partially visible 
in figures 3(c) and (d). The structures overlap one another and are connected by their bodies at the edges (the extent of the connection between structures naturally depends on the chosen isosurface). It is unknown whether the observed regular annular banding of the isosurface is a real, coherent signal or the result of the isosurface rendering of noise in the data. A movie showing the full dynamics is available as supplementary movie 2 .

\subsection{Spatio-temporal behaviour}

Figure 4 shows the spatio-temporal behaviour of the CHW. Slices of the vorticity field (figure 4(a)) and density field (figure 4(b)) taken at the mean positions of the respective interfaces $(z=-0.11$ for the velocity interface, $z=-0.22$ for the density interface) are stacked in time to form a spatio-temporal (Hovmöller) diagram.

The wave propagates mostly leftwards in both fields, i.e. with phase speed $c<0$. The coupling of the fields is confirmed, as both travel at the same instantaneous phase speed and undergo the same gradual change in wavelength. The range of wavelengths observed from this figure is $\lambda \in[3.5,6.0]$, corresponding to a range in wavenumber $k \in$ $[1.05,1.80]$. The range of phase speeds, as determined by the slope of the characteristics, is $c \in[-0.18,0.05]$.

There is evidence of a preferred wavelength, as splitting events occur when the wavelength becomes 'too large', presumably due to wave stretching (e.g., at $t \approx 180$ and $t \approx 270$ ), consistent with some development in $x$ of the mean flow (Tedford et al. 2009; Carpenter et al. 2010). This development of the mean flow along $x$ is an inevitable consequence of the convective acceleration of the flow as it travels along the duct. In experiments for which gravity forcing dominates over the longitudinal pressure gradient $\left(\theta \gtrsim \tan ^{-1}(H / L) \approx 2^{\circ}\right.$, as in the present case) this effect is small and the flow is nearly parallel. Splitting events require a difference in phase speed between the parent and daughter structures (stretching-splitting) and we indeed observe for a short time a daughter wave propagating with slightly positive phase speed while the parent continues at unchanged, negative phase speed (at $x \approx-10, t \approx 180$ and $x \approx-13, t \approx 270$ in figure 4).

\subsection{Phase-averaged properties}

To characterise the 'typical' structure of the CHW and reduce its spatio-temporal complexity, we phase-average the flow variables over a single wavelength by following the wave along a characteristic, an operation denoted by $\langle\cdot\rangle$. To make this calculation, we determine the phase speed $c$ and wavelength $\lambda$ by considering the interval $t \in[300,335]$ at the end of the experiment. This period, indicated by solid horizontal lines in figures $4(\mathrm{a})$ and (b), was selected as it is during this period that the wave has reached a very nearly constant negative phase speed and a steady wavelength, making the analysis easier and more meaningful. We determine the phase speed $c=-0.078$ and wavelength $\lambda=4.30$ (wavenumber $k=1.46$ ) by fitting in time the location of the vorticity minimum contained within the wavelength denoted by two thick solid sloping lines in figures 4(a) and (b). The dashed sloping lines having slope $c$ and spacing $\lambda$, drawn half a wavelength from the solid lines, extend over the entire spatio-temporal plot and demonstrate that the phase-averaged properties are representative of the flow at earlier times.

The three-dimensional structure of the resulting phase-averaged vorticity in the region $x \in[0,4.30]$ is shown in figure $4(\mathrm{c})$ and (d), using the same isosurface level $\left\langle\omega_{y}\right\rangle=-2.5$ as in figure 3. For better visualisation, this region has been replicated to $x \in[4.30,8.60])$ to show two wavelengths for the structure. The two panels show views from different angles. Naturally, we recover the features identified in figure 3, but are able to discern 

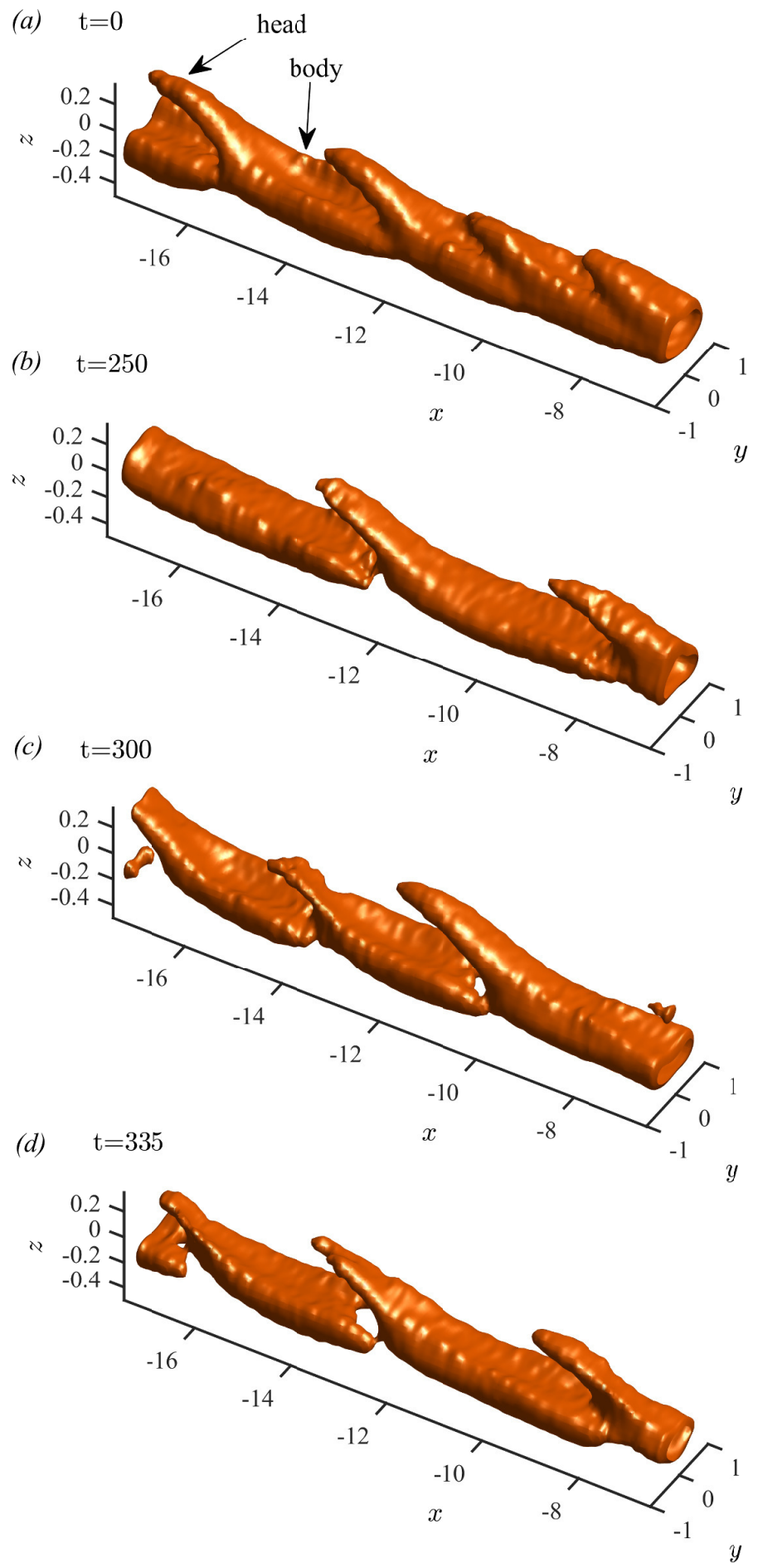

Figure 3. Isosurfaces of spanwise vorticity $\omega_{y}=-2.5$ at the same times as in figure 2 . The $z$ axis is stretched by a factor of 3 and only $z \in[-0.5,0.3]$ is shown. 
(a)

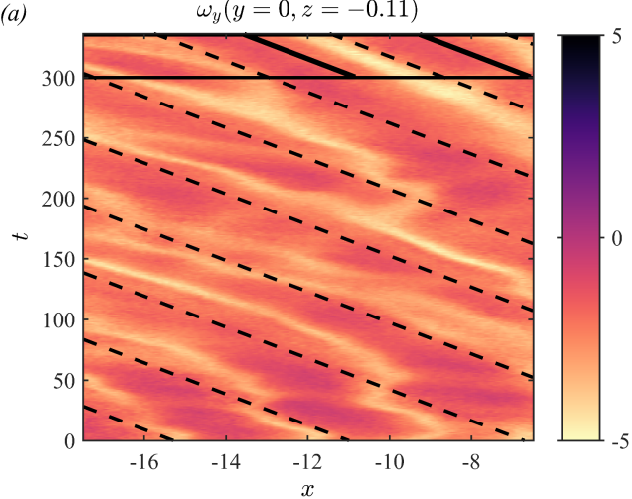

(c)

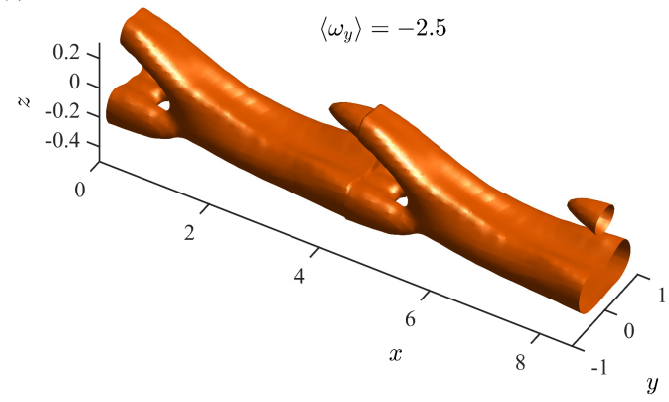

(b)

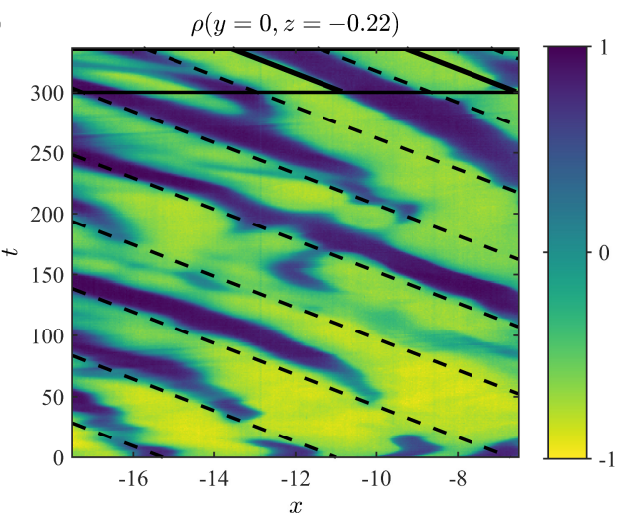

(d)

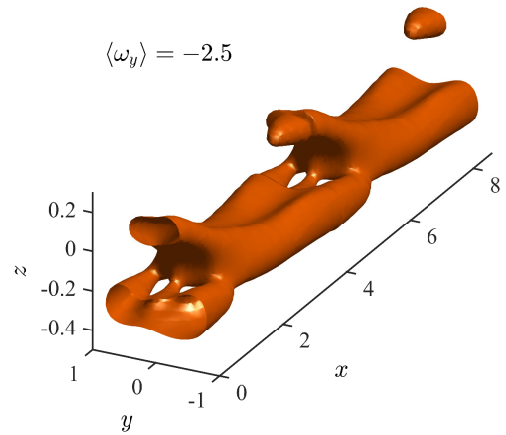

Figure 4. (a-b) Spatio-temporal diagrams taken in the vertical mid-plane and horizontal interfacial plane: (a) $\omega_{y}(y=0, z=-0.11)$ and (b) $\rho(y=0, z=-0.22)$. - - - characteristics with phase speed $\mathrm{c}=-0.078$ and wavelength $\lambda=2 \pi / 1.46=4.3$. - Start $(t=300)$ and end $(t=335)$ of the phase averaging window, represented by - (c)-(d) Two views of the isosurface of phase-averaged spanwise vorticity $\left\langle\omega_{y}\right\rangle=-2.5$. Two wavelengths are shown by repeating the structure along $x$. Note the slight lack of periodicity evidenced by the discontinuity at $x=4.30$. The $z$ axis is stretched by a factor of 3 and only $z \in[-0.5,0.3]$ is shown.

further details. The structure is remarkably symmetrical about $y=0$ and very nearly periodic (see the small discontinuity at $x=\lambda=4.30$ where the replicated structures join), despite the slow stretching in $x$ discussed above. It is also triply connected, and its tilt with respect to $x$ (the duct axis) is $10^{\circ}$ (maximum angle at the inflection point).

We discuss further details of the phase averaged structure of the $\mathrm{CHW}$ (including the other flow variables $\rho, u, v, w)$ in $\S 6$ when comparing it with the linear confined Holmboe instability $(\mathrm{CHI})$ predicted by the stability analysis introduced in the next section $\S 5$.

\section{Linear stability: formulation}

In this section, we derive the eigenvalue problem describing the linear stability of the experimental flow from the governing equations. We model the flow by the incompressible Navier-Stokes equations under the Boussinesq approximation. The non-dimensional equations of motion under the notation and conventions adopted in $\S 3.2$ are

$$
\begin{aligned}
\boldsymbol{\nabla} \cdot \mathbf{u} & =0, \\
\partial_{t} \mathbf{u}+\mathbf{u} \cdot \boldsymbol{\nabla u} & =-\boldsymbol{\nabla} p+\operatorname{Ri}(-\cos \theta \hat{\mathbf{z}}+\sin \theta \hat{\mathbf{x}}) \rho+R^{-1} \nabla^{2} \mathbf{u}, \\
\partial_{t} \rho+\mathbf{u} \cdot \boldsymbol{\nabla} \rho & =(\operatorname{Re} S c)^{-1} \nabla^{2} \rho,
\end{aligned}
$$


where, for the experiment considered, $\theta=5^{\circ}, R e=440, R i=0.25, S c=700$ (see $\S 3.4 .1$.

We first consider the general case of three-dimensional perturbations on a twodimensional base flow $(3 \mathrm{P}-2 \mathrm{~B})$ in $\S 5.1$ before showing how the equations can be simplified to the more commonly-used study of two-dimensional perturbations on a one-dimensional base flow (2P-1B) in $\S 5.2$. We discuss our choice of base flow in $\S 5.3$.

\subsection{Three-dimensional perturbations, two-dimensional base flow (3P-2B)}

We assume a steady parallel two-dimensional base flow $\mathbf{U} \equiv[U(y, z), 0,0]$ and onedimensional density distribution $R(z)$ (note that (5.1) do not support steady states with this base flow and $R(y, z)$ ). We superimpose three-dimensional infinitesimal perturbations, so that the full velocity field is

$$
\mathbf{u} \equiv\left[\begin{array}{c}
U(y, z) \\
0 \\
0
\end{array}\right]+\varepsilon\left[\begin{array}{c}
u^{\prime}(x, y, z, t) \\
v^{\prime}(x, y, z, t) \\
w^{\prime}(x, y, z, t)
\end{array}\right]
$$

and similarly $\rho \equiv R(z)+\varepsilon \rho^{\prime}(x, y, z, t), p \equiv P(z)+\varepsilon p^{\prime}(x, y, z, t),(|\varepsilon| \ll 1)$. We assume that each perturbation variable $\psi^{\prime}$ has periodic wavelike behaviour in $x$ and $t$ :

$$
\psi^{\prime}(x, y, z, t) \equiv \hat{\psi}(y, z) \exp (i k x+\sigma t),
$$

where the real part is implied. Note the fundamental distinction between this $3 \mathrm{P}-2 \mathrm{~B}$ form and the $2.5 \mathrm{P}-1 \mathrm{~B}$ form in $(2.2)$. We tackle the temporal stability problem by specifying the perturbation wavelength $k>0 \in \mathbb{R}$ and solve the following eigenvalue problem for the (temporal) growth rate $\sigma \in \mathbb{C}$ and eigenfunctions $\hat{\psi}(y, z) \in \mathbb{C}$ :

$$
\sigma\left[\begin{array}{ccc}
\nabla^{2} & & \\
& \nabla^{2} & \\
& & \mathcal{I}
\end{array}\right]\left[\begin{array}{c}
\hat{v} \\
\hat{w} \\
\hat{\rho}
\end{array}\right]=\left[\begin{array}{ccc}
\mathcal{L}_{v} & \mathcal{L}_{v w} & \mathcal{L}_{v \rho} \\
\mathcal{L}_{w v} & \mathcal{L}_{w} & \mathcal{L}_{w \rho} \\
& \mathcal{L}_{\rho w} & \mathcal{L}_{\rho}
\end{array}\right]\left[\begin{array}{c}
\hat{v} \\
\hat{w} \\
\hat{\rho}
\end{array}\right],
$$

where

$$
\begin{aligned}
\mathcal{L}_{v} & \equiv-i k U \nabla^{2}+i k\left(\partial_{y y} U-\partial_{z z} U\right)-2 i k \partial_{z} U \partial_{z}+R e^{-1} \nabla^{4}, \\
\mathcal{L}_{w} & \equiv-i k U \nabla^{2}+i k\left(\partial_{z z} U-\partial_{y y} U\right)-2 i k \partial_{y} U \partial_{y}+R e^{-1} \nabla^{4}, \\
\mathcal{L}_{v w} & \equiv 2 i k\left(\partial_{y z} U+\partial_{z} U \partial_{y}\right) \\
\mathcal{L}_{w v} & \equiv 2 i k\left(\partial_{y z} U+\partial_{y} U \partial_{z}\right) \\
\mathcal{L}_{\rho} & \equiv-i k U+(R e S c)^{-1} \nabla^{2} \\
\mathcal{L}_{v \rho} & \equiv R i\left(\cos \theta \partial_{y z}-i k \sin \theta \partial_{y}\right), \\
\mathcal{L}_{w \rho} & \equiv R i\left\{\cos \theta\left(\partial_{z z}-\nabla^{2}\right)-i k \sin \theta \partial_{z}\right\} \\
\mathcal{L}_{\rho w} & \equiv-\partial_{z} R .
\end{aligned}
$$

Here $\mathcal{I}$ is the identity, $\nabla^{2} \equiv-k^{2}+\partial_{y y}+\partial_{z z}$ and $\nabla^{4} \equiv k^{4}+\partial_{y y y y}+\partial_{z z z z}+2 \partial_{y y} \partial_{z z}-$ $2 k^{2}\left(\partial_{y y}+\partial_{z z}\right)$. The streamwise velocity eigenfunction is then deduced as

$$
\hat{u}=-\frac{i}{k}\left(\partial_{y} \hat{v}+\partial_{z} \hat{w}\right) .
$$

The derivation of (5.4)-(5.6) is given in appendix A.

We represent the rigid, impermeable walls of the duct by imposing no-slip boundary conditions for velocity perturbations: $\hat{v}=\hat{w}=0$ along $y, z= \pm 1$. To impose $\hat{u}=0$ along $y, z= \pm 1,(5.6)$ requires that we further impose $\partial_{y} \hat{v}=0$ along $y= \pm 1$ and $\partial_{z} \hat{w}=0$ along $z= \pm 1$. Physically, no salt diffuses through the walls of the duct, hence the boundary 
conditions for the density perturbation are $\partial_{y} \hat{\rho}=0$ along $y= \pm 1$ and $\partial_{z} \hat{\rho}=0$ along $z= \pm 1$.

We solve the eigenvalue problem (5.4) numerically, using second-order finite-difference discretisation with uniform spacing in both $y$ and $z$. A resolution of $120 \times 120$ proved to be sufficient for convergence of the results, and required solving a $\left(3 \times 118^{2}\right) \times\left(3 \times 118^{2}\right)$ eigenvalue problem. The results are presented in $\S 6.1$.

\subsection{Two-dimensional perturbations, one-dimensional base flow (2P-1B)}

To contextualise the results of the formulation introduced above, we will also discuss the results of the more commonly used $2 \mathrm{P}-1 \mathrm{~B}$ analysis. This represents the special case for which the spanwise velocity perturbation $v$ and all variations in the spanwise direction $y$ are ignored, so that the full velocity field is

$$
\mathbf{u}_{\dagger} \equiv\left[\begin{array}{c}
U(z) \\
0 \\
0
\end{array}\right]+\varepsilon\left[\begin{array}{c}
u_{\dagger}^{\prime}(x, z, t) \\
0 \\
w_{\dagger}^{\prime}(x, z, t)
\end{array}\right],
$$

and $\rho_{\dagger} \equiv R(z)+\varepsilon \rho_{\dagger}^{\prime}(x, z, t), p_{\dagger} \equiv P(z)+\varepsilon p_{\dagger}^{\prime}(x, z, t)$, with all perturbations

$$
\psi_{\dagger}^{\prime}(x, z, t) \equiv \hat{\psi}_{\dagger}(z) \exp (i k x+\sigma t) .
$$

Note that this $2 \mathrm{P}-1 \mathrm{~B}$ form corresponds to the special case $\beta=0$ of the $2.5-1 \mathrm{~B}$ form in (2.2). Here and below, the subscript/superscript $\dagger$ distinguishes the variables and operators of the $2 \mathrm{P}-1 \mathrm{~B}$ analysis from those of the $3 \mathrm{P}-2 \mathrm{~B}$ analysis.

The governing equations for this problem are obtained from (5.4)-(5.6) by removing $\hat{v}$ and setting $\partial_{y}=0$, i.e.

$$
\sigma\left[\begin{array}{ll}
\nabla_{\dagger}^{2} & \\
& \mathcal{I}
\end{array}\right]\left[\begin{array}{c}
\hat{w}_{\dagger} \\
\hat{\rho}_{\dagger}
\end{array}\right]=\left[\begin{array}{cc}
\mathcal{L}_{w}^{\dagger} & \mathcal{L}_{w \rho}^{\dagger} \\
\mathcal{L}_{\rho w}^{\dagger} & \mathcal{L}_{\rho}^{\dagger}
\end{array}\right]\left[\begin{array}{c}
\hat{w}_{\dagger} \\
\hat{\rho}_{\dagger}
\end{array}\right]
$$

where

$$
\begin{aligned}
\mathcal{L}_{w}^{\dagger} & \equiv-i k U \nabla_{\dagger}^{2}+i k \partial_{z z} U+R e^{-1} \nabla_{\dagger}^{4}, \\
\mathcal{L}_{\rho}^{\dagger} & \equiv-i k U+(R e S c)^{-1} \nabla_{\dagger}^{2}, \\
\mathcal{L}_{w \rho}^{\dagger} & \equiv R i\left\{\cos \theta\left(\partial_{z z}-\nabla_{\dagger}^{2}\right)-i k \sin \theta \partial_{z}\right\}, \\
\mathcal{L}_{\rho w}^{\dagger} & \equiv-\partial_{z} R,
\end{aligned}
$$

and $\nabla_{\dagger}^{2} \equiv-k^{2}+\partial_{z z}, \nabla_{\dagger}^{4} \equiv k^{4}+\partial_{z z z z}-2 k^{2}+\partial_{z z}$. The streamwise velocity eigenfunction is simply $\hat{u}_{\dagger}=-(i / k) \partial_{z} \hat{w}_{\dagger}$. As in $\S 5.1$, we discretise (5.9) in $z$ using second-order finite differences and the boundary conditions at $z= \pm 1$ are no-slip for $\hat{w}_{\dagger}, \hat{u}_{\dagger}: \hat{w}_{\dagger}=\partial_{z} \hat{w}_{\dagger}=0$ and no-flux for $\hat{\rho}_{\dagger}: \partial_{z} \hat{\rho}_{\dagger}=0$. The results are presented in $\S 6.2$.

\subsection{Experimental base flow}

In order to obtain linear stability predictions which are most relevant for comparison with the CHW observed in the experiment, we use averages of the experimental flow. To obtain a representative, canonical $U(y, z)$ for the $3 \mathrm{P}-2 \mathrm{~B}$ analysis, the streamwise velocity $u$ was first averaged along $x$ and over 50 ATU before the start of the phase averaging window, i.e. for $t \in[250,300]$, and symmetrised about $y=0: U(y, z) \equiv\left[\langle u\rangle_{x, t}(y, z)+\right.$ $\left.\langle u\rangle_{x, t}(-y, z)\right] / 2$ (figure 5(a)). The profile used for the $2 \mathrm{P}-1 \mathrm{~B}$ analysis is $U(z) \equiv U(y=$ $0, z)$ (figure $5(\mathrm{~b})$ ). Note that since the flow is close to steady, the duration of 50 ATU 

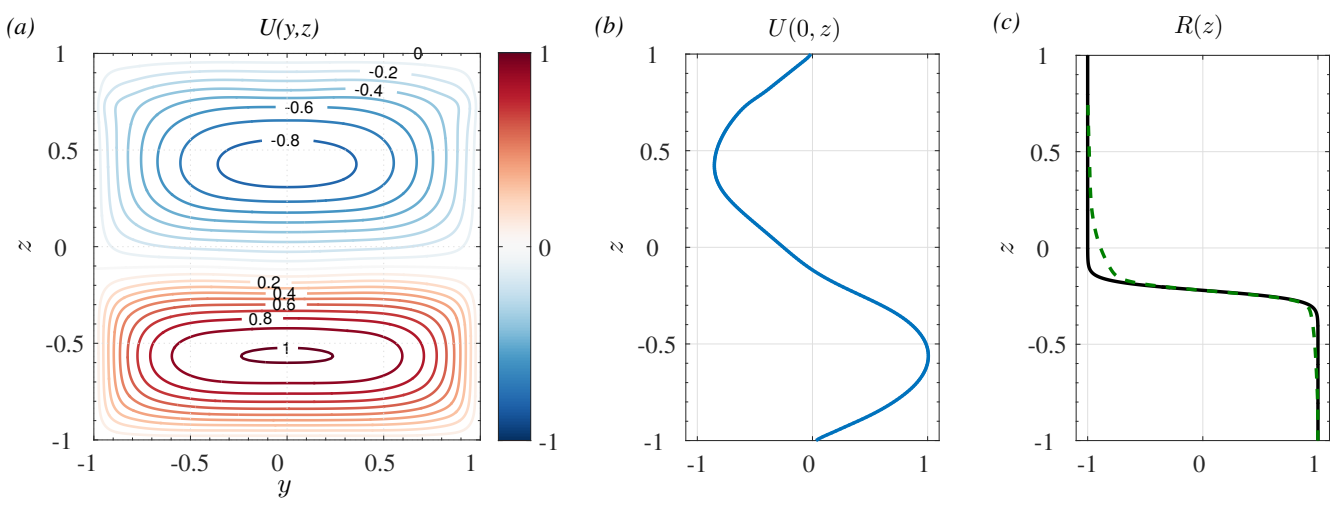

Figure 5. Base flow for stability analysis using experimental flow averaged over $t \in[250,300]$ before the phase average window. (a) Streamwise velocity $U(y, z)$ for the $3 \mathrm{P}-2 \mathrm{~B}$ analysis. (b) $U(z)=U(y=0, z)$ for the $2 \mathrm{P}-1 \mathrm{~B}$ analysis. Density: - - $\langle\rho\rangle_{x, y, t}$ conditioned at the interface, and $-R(z)$ the tanh fit with the mixed layer removed as used for the $3 \mathrm{P}-2 \mathrm{~B}$ and $2 \mathrm{P}-1 \mathrm{P}$ analyses (see text for details).

for the phase averaging window is arbitrary and the results were only weakly sensitive to this choice.

The measured density field was averaged over the same $x$ and $t$ windows, with an additional averaging over $y \in[-1,1]$ since only the one-dimensional base density profile $R(z)$ enters the stability problem. Being distorted by a finite amplitude HW, the interface does not lie on $z=$ const. Since the linear stability properties of HIs are known to be sensitive to the thickness of the interface, it is important to ensure that $R(z)$ has a thickness representative of undistorted local profiles. We achieve this by conditionally averaging over $x$ and $y$, i.e. shifting all profiles vertically in order to align them at the interface before averaging them (shown in dashed green in figure $5(\mathrm{c})$. Finally, our aim is to elucidate the origin of CHWs (i.e. to identify and understand the underlying physical mechanisms leading to their observed behaviour) and not to capture either the initial transient of the experiment or the nonlinear wisp ejection dynamics, which presumably produces the slightly mixed region in the region $-0.2 \lesssim z \lesssim 0.1$ described in $\S 4.1$. We therefore eliminate this mixed region by fitting a hyperbolic tangent function (shown in solid black) to the lower (unmixed) region $(z \leqslant-0.2)$ of the previously obtained conditional average. The best-fit base profile we use for the $3 \mathrm{P}-2 \mathrm{~B}$ and $2 \mathrm{P}-1 \mathrm{~B}$ analyses is $R(z) \equiv-\tanh \left\{\left(z-z_{0}\right) / \delta\right\}$ with $z_{0}=-0.22$ and $\delta=0.047$.

\section{Linear stability: predictions, comparison and discussion}

\subsection{P-2B results}

\subsubsection{Dispersion relation}

The dispersion relation $\sigma(k)$ where $\sigma \equiv \sigma_{r}+i \sigma_{i}$ (growth rate $\sigma_{r}$ and phase speed $\left.c \equiv-\sigma_{i} / k\right)$ is shown for growing modes $\sigma_{r}>0$ only in figure 6 (thick blue curve). We observe a lower wavenumber band of positive growth rate signalling an unstable mode for the range of wavenumbers $k \in[0.8,2.2]$, as well as another band with lower growth rate for $k \in[2.3,5.0]$. The fastest growing mode (with largest $\sigma_{r}$ ) is predicted to occur at $k=1.32$ with growth rate $\sigma_{r}=0.0824$ and negative phase speed $c=-0.213$. The other mode of instability has maximum growth rate of $\sigma_{r}=0.0192$ and positive phase speed $c=0.55$. As discussed in $\S 2$, the existence of a faster and a slower growing Holmboe modes with different phase speed magnitudes, growth rates and wavenumbers is typical of asymmetric 

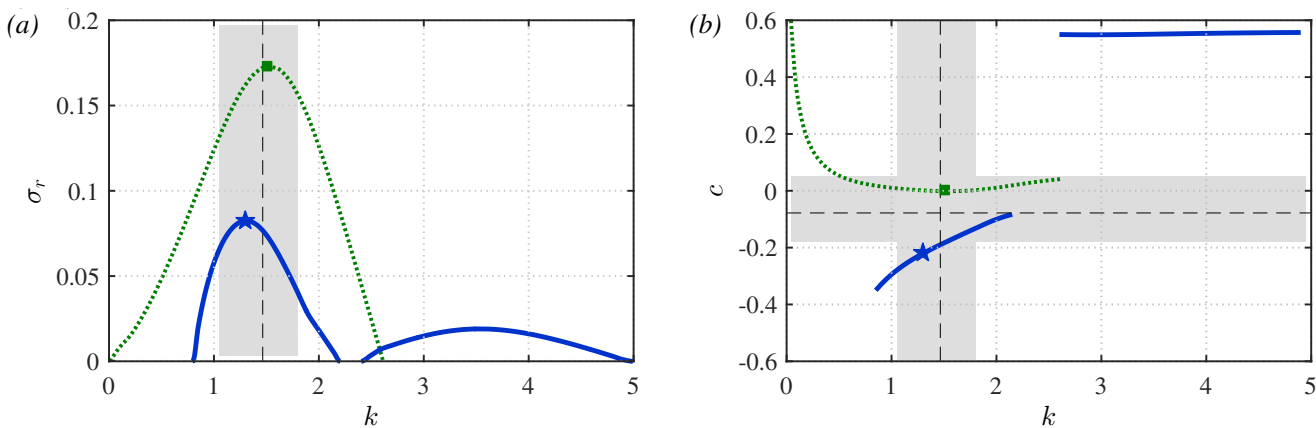

Figure 6. Dispersion relation: (a) positive growth rate $\sigma_{r}$ and (b) phase speed $c$. $-3 \mathrm{P}-2 \mathrm{~B}$ analysis and $\cdots 2$ 2P-1B analysis. ( $\star$ and - mark the respective theoretically predicted fastest growing modes.) Experimental range observed and - - - values used for the phase average.

profiles (where the relatively sharp density interface and midpoint of the shear layer are not coincident). In the following we will focus on the faster growing, leftward propagating band of instability, which we identify as a confined Holmboe instability (CHI).

The ranges of wavenumbers and phase speeds observed in the experimental CHW (figure 4(a)-(b)) are shown as grey shading: $k \in[1.05,1.80], c \in[-0.18,0.05]$, and the phase average values $(k=1.46, c=-0.078)$ as black dashed lines. The wavenumber of the fastest growing mode compares well with the experimental values, and with the phase averaged structure $(k=1.32$ vs $k=1.46)$. The agreement in phase speed is qualitatively good overall, despite the fastest growing mode travelling significantly faster (in magnitude) than the representative phase average value $(c=-0.213$ vs $c=-0.078)$. There is overlap between the unstable branch and the intersection of the two perpendicular grey boxes in figure $6(\mathrm{~b})$, i.e. the shortest observed CHWs having $k \in[1.5,1.8]$ and moving at the highest speeds $c \in[-0.18,-0.15]$ are consistent with the slower-growing modes of the CHI.

A variety of causes can be invoked for the discrepancy in phase speed:

(i) The base velocity (i.e. with $\partial_{x} U(y, z)=0$ ) is only an approximation to the slowly developing, non-parallel experimental flow, and such streamwise variation naturally affects the wavelength and speed of HWs.

(ii) The base flow used in the stability calculation is a spatio-temporal average from the unsteady experimental flow that contains the wave. The most obvious effects of this wave on the density profile have been partially eliminated in determining $R(z)$, but not in determining $U(y, z)$. It is not a priori obvious that this intuitive choice of base flow should correctly predict the linear instability that grows to finite amplitude and hence is observed in the experimental flow.

(iii) The experimental flow is not the result of an initial value problem. The nonlinear wave state selected by the flow does not have to result from the saturation of a monochromatic instability (the fastest growing mode), and there is therefore no reason to think that the nonlinear phase speed should match closely the phase speed of the fastest growing mode.

To summarise, the analysis of the dispersion relation reveals reasonably good agreement between the predicted $\mathrm{CHI}$ and the observed $\mathrm{CHW}$, despite the challenge of inferring the nonlinear properties of our flow from a linear analysis. In the next section we focus on comparing the three-dimensional structure of the fastest growing $\mathrm{CHI}$ with the $\mathrm{CHW}$. 
(a)

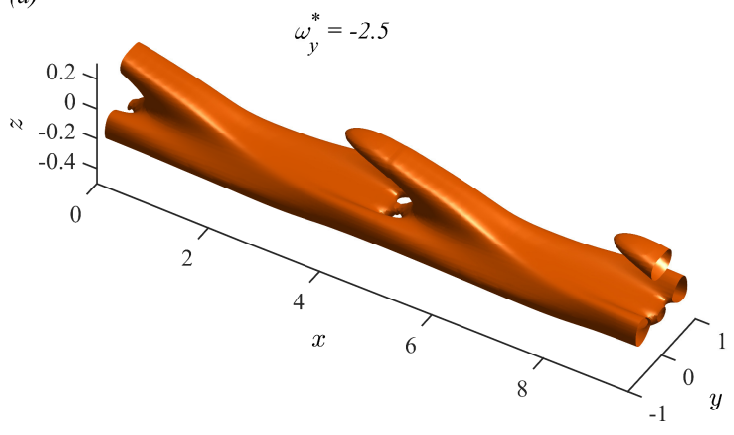

(b)

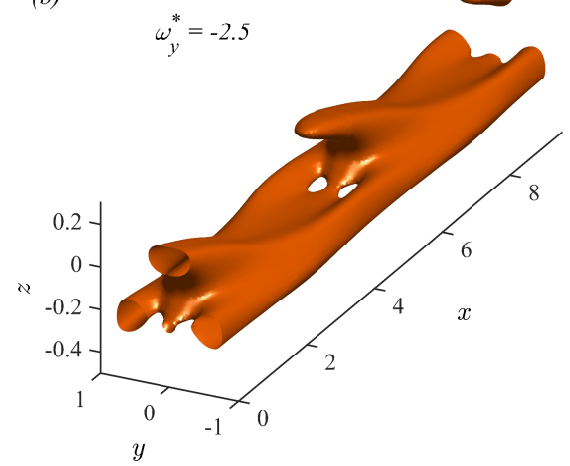

FiguRE 7. Fastest growing mode of the CHI: isosurface $\omega_{y}^{*}=-2.5$ (see (6.1) for definition of $\omega_{y}^{*}$ ). We show a double wavelength under two different views (a) and (b) for direct comparison with figures $4(\mathrm{c})$ and (d), respectively. As in those figures, the $z$-axis is stretched by a factor of 3 and only $z \in[-0.5,0.3]$ is shown for clearer visualisation.

\subsubsection{Eigenfunctions}

We turn to the three-dimensional structure of the eigenfunction of the fastest growing CHI. To provide meaningful comparison with experimental data, the vorticity eigenfunction is added to the base flow vorticity

$$
\omega_{y}^{*} \equiv \partial_{z} U+\alpha \operatorname{Re}\left\{\hat{\omega}_{y}(y, z) \exp (i k x+\phi)\right\},
$$

where the superscript ${ }^{*}$ denotes this reconstruction and $\alpha, \phi$ are constants to be determined. The phase parameter $\phi$ was simply chosen to compare directly with the phase of figure 4. The scaling parameter $\alpha$ contains the information relative to the finite amplitude of the wave observed in the experiment. It was determined by imposing the condition that the volume-averaged root mean square (rms) of the wave perturbation in the reconstructed vorticity $\omega_{y}^{*}-\partial_{z} U$ is equal to that of the wave perturbation in the phase average vorticity $\left\langle\omega_{y}\right\rangle-\partial_{z} U$ :

$$
\left\langle\left(\omega_{y}^{*}-\partial_{z} U\right)^{\mathrm{rms}}\right\rangle_{x, y, z}=\frac{\alpha}{2} \sqrt{\left\langle\hat{\omega}_{y}^{2}\right\rangle_{y, z}}=\left\langle\left(\left\langle\omega_{y}\right\rangle-\partial_{z} U\right)^{\mathrm{rms}}\right\rangle_{x, y, z}=0.31,
$$

where rms denotes the operator $\psi^{\mathrm{rms}}(x, y, z) \equiv \sqrt{\left(\psi-\langle\psi\rangle_{x, y, z}\right)^{2}}$. The first equality is a simple consequence of the definition in (6.1), the second equality is our condition and the third is the experimentally measured value.

The isosurface $\omega_{y}^{*}=-2.5$ of the resulting reconstruction for two wavelengths is shown in figure 7 . A direct comparison with the phase average $\left\langle\omega_{y}\right\rangle=-2.5$ of figures $4(\mathrm{c})$ and $4(\mathrm{~d})$ reveals excellent agreement between the three-dimensional structure of the $\mathrm{CHI}$ and the CHW. The tilt of the structure with respect to the duct axis is $8^{\circ}$ (maximum angle at the inflection point), slightly smaller than the $10^{\circ}$ obtained from the experimental phase average. The general geometry, including the detail of the triple connectedness of the head-to-tail connection, is faithfully reproduced. Supplementary movie 3 offers a panoramic visualisation of the vorticity isosurface of the $\mathrm{CHW}$ and $\mathrm{CHI}$, allowing for more detailed comparison.

To reveal the structure of the wave further and to allow for more detailed comparison, we build, by analogy with (6.1), a more complete picture of the wave field by considering 
the following variables associated with the fastest growing mode:

$$
\begin{array}{rr}
\rho^{*} \equiv R(z)+\alpha \operatorname{Re}\{\hat{\rho}(y, z) \exp (i k x+\phi)\}, \\
u^{*} \equiv U(y, z)+\alpha \operatorname{Re}\{\hat{u}(y, z) \exp (i k x+\phi)\}, \\
v^{*} \equiv \\
w^{*} \equiv & \alpha \operatorname{Re}\{\hat{v}(y, z) \exp (i k x+\phi)\}, \\
& \alpha \operatorname{Re}\{\hat{w}(y, z) \exp (i k x+\phi)\},
\end{array}
$$

using the previously determined value of $\alpha$. We plot these wave variables in planar cuts in figure 8 (experimentally observed $\mathrm{CHW}$ ) and figure 9 (theoretically predicted fastest growing CHI). These figures combine views in the vertical midplane $y=0$, horizontal interfacial planes ( $z=-0.22$ and $z=-0.11$ for density and velocity, respectively) and one cross-sectional plane for $\omega_{y}, \rho, u, v, w$ for a single wavelength.

There is good agreement between the observed and predicted structure in each of these variables. Even though only the magnitude of the perturbation vorticity field was matched with the experimental value, the other variables in figure 9 have amplitudes very close to those in figure 8 (colour bars have the same limits in both figures and black contours are the same). The spanwise and vertical velocities $v$ and $w$ are small relative to the streamwise velocity, with a maximum of about $6 \%$ of the maximum of $u$. The quantitative and qualitative agreement extends to a number of interesting features as follows.

(i) The sheared vertical pattern in $w$, typical of Holmboe modes, is clearly identifiable in panels $m$ of figures 8 and 9 .

(ii) The horizontal chevron pattern identified earlier in the three-dimensional visualisation of the vorticity $\omega_{y}$ is again visible in panels $b$ (highlighted by the contours). It is also found in the streamwise velocity $u$ (panels $h$ ), where it contributes the main signal in this interfacial plane where the mean velocity is zero.

(iii) The relative phase of the density $\rho$ and vorticity $\omega_{y}$ reveals that the 'head' of the vorticity structure is shifted slightly left of the top of the upward-pointing density cusp, while its 'body' appears to rest on the sloping interface of the cusp for which $\partial_{x} \rho<0$ (panels $a, b, d, e$ ). This gradient is responsible for the baroclinic production of negative vorticity (reinforcing the negative mean shear). The density crests and troughs have a distinct convex structure (their vertical displacement peaks in the middle of the duct $y=0$ and decays near the boundaries $y= \pm 1$ ), making the gradients $\partial_{x} \rho$ larger in the middle of the duct, presumably responsible for confinement of the vorticity and the nonlinear maintenance of the CHW at finite amplitude.

(iv) The variables clearly have different symmetries about the midplane $y=0$, as is apparent in panels $e, h, k$, and $n$. The structure of the CHI correctly predicts that the density $\hat{\rho}$, streamwise velocity perturbation $\hat{u}$ and vertical velocity perturbation $\hat{w}$ are even functions about this midplane, while the spanwise velocity perturbation $\hat{v}$ is an odd function, implying that the $\mathrm{CHI}$ is of varicose rather than sinuous form. It is important to stress that we did not impose these symmetries; they arose naturally as solutions to the $3 \mathrm{P}-2 \mathrm{~B}$ stability eigenvalue problem. Furthermore, and perhaps unsurprisingly, the $2.5 \mathrm{P}-1 \mathrm{~B}$ description of a normal mode travelling at an angle as described in $\S 2.3 .3$ is not appropriate to describe this structure, as the spanwise periodicity of $\hat{v}$ is clearly different from the other perturbation quantities $\hat{u}, \hat{w}$ and $\hat{\rho}$. The odd symmetry of $\hat{v}$ seems to be inherently related (for reasons which are not yet fully understood) to the spanwise boundary conditions $\hat{v}=\partial_{y} \hat{v}=0$ imposed by incompressibility at the duct walls at $y= \pm 1$. Indeed, a very similar eigenstructure was observed when we solved the $3 \mathrm{P}-2 \mathrm{~B}$ problem with these boundary conditions for the spanwise uniform base flow $U(z)$. (For reasons of brevity we do not discuss this problem further here.) 

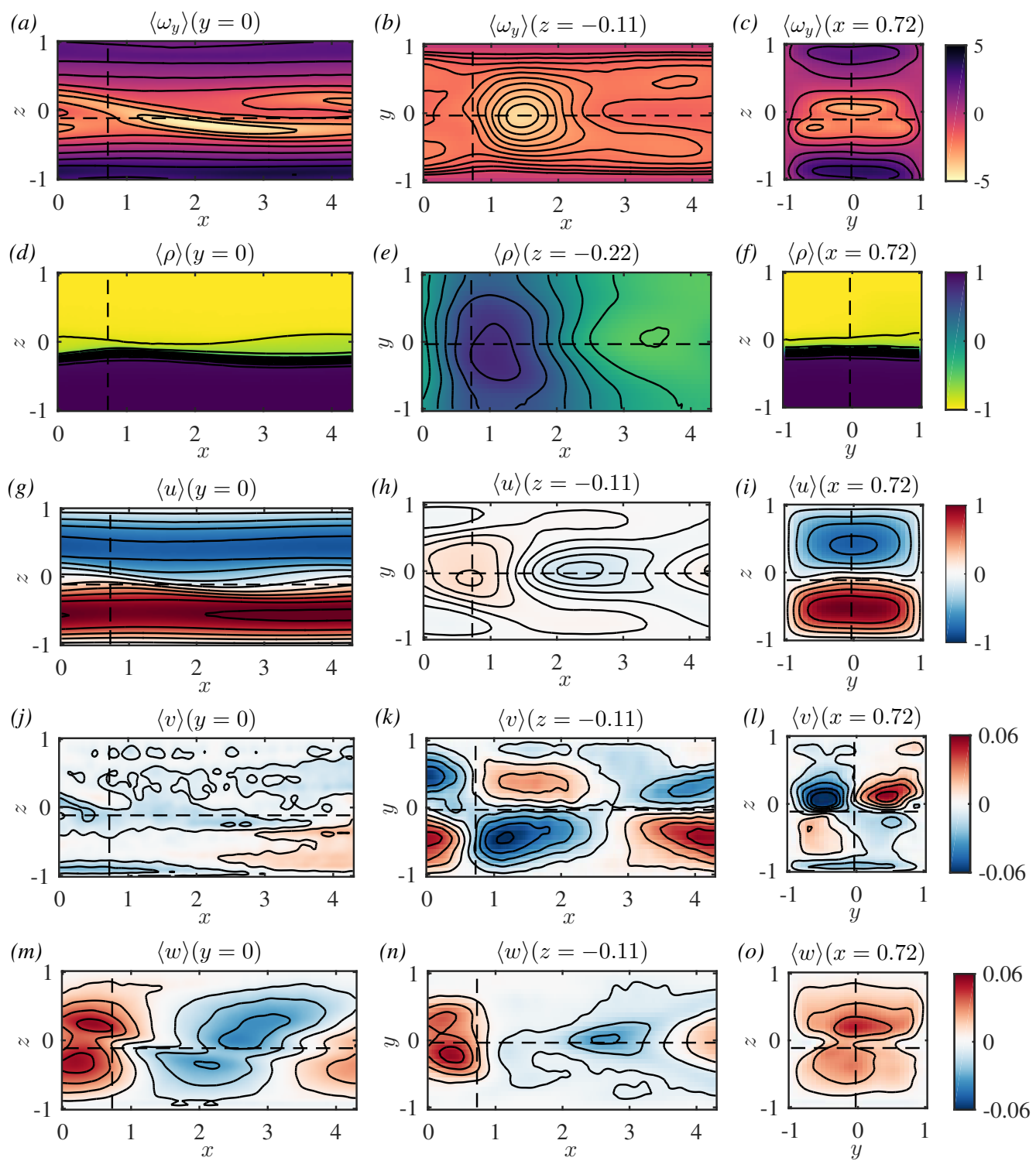

Figure 8. Experimental phase average spanwise vorticity $\left\langle\omega_{y}\right\rangle$ (a-c), density $\langle\rho\rangle$ (d-f), and velocity components $\langle u\rangle$ (g-i), $\langle v\rangle(\mathrm{j}-1),\langle w\rangle(\mathrm{m}-\mathrm{o})$. The panels show the vertical mid-plane $y=0$ (left column), horizontal interfacial plane ( $z=-0.11$ for velocity and $z=-0.22$ for density) (middle column) and a cross-sectional plane $x=0.72$ (right column). The colour bars are the same for a given variable. Ten black contours equally spaced across the range of the colour bar are also shown, except for $(\mathrm{b})$ and $(\mathrm{h})$ where the ten contours are across $[-4,-1]$ and $[-0.25,0.25]$ respectively, to highlight features of interest. Dashed lines represent the planes in the other panels.

(v) A consequence of the specific symmetry of the CHW/CHI is the horizontal, inplane divergence and convergence of $v$ (panels $k$ and $l$ ) around the upward density crest (panels $e$ and $f$ ). Due to the relatively large stratification $(R i=1 / 4)$, the horizontal flow around the density crest is energetically preferred to the vertical flow above it. The convex structure of the density crest allows the flow to move around it horizontally. An 

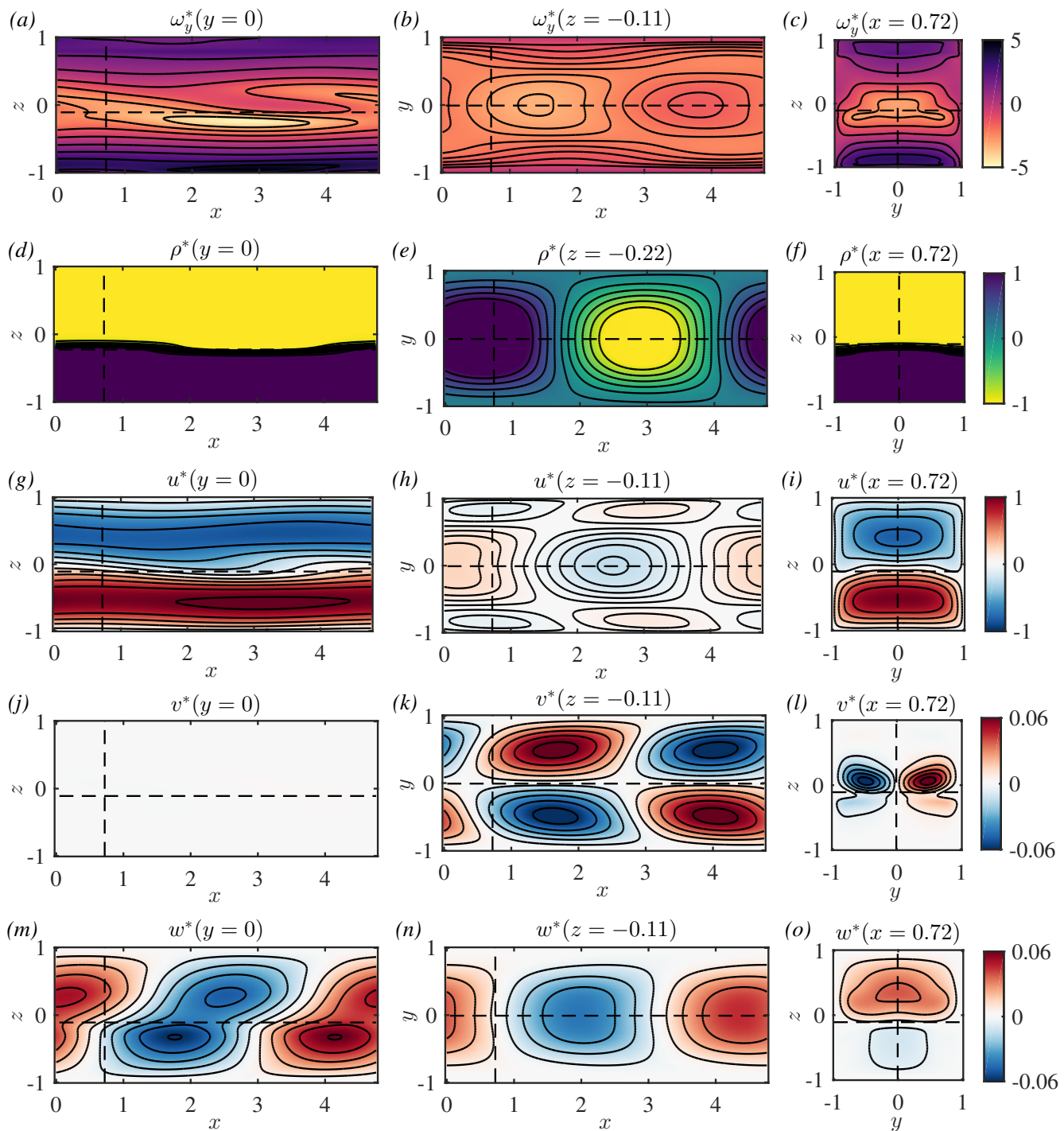

Figure 9. Fastest growing CHI of the 3P-2B linear stability analysis superimposed on the base flow (see (6.1), (6.2) and (6.3) for definitions of the variables). All panels plotted as in figure 8 for direct comparison.

important consequence is that it gives rise to relatively large spanwise gradients $\left|\partial_{y} v\right|$, positive in the centre of the duct $y \in[-0.5,0.5]$ and negative near the boundaries $|y| \in$ $[0.5,1]$. These gradients have a vortex stretching effect on $\omega_{y}$ through the term $\omega_{y} \partial_{y} v$, producing negative vorticity in the centre (reinforcing the mean shear), and positive vorticity near the boundaries (weakening the mean shear). They could play an important role in explaining the spanwise confinement (largest values of $\left|\omega_{y}\right|$ in the centre) and maintenance of the $\mathrm{CHW}$ at finite amplitude. 


\section{2. $2 P-1 B$ results}

\subsubsection{Dispersion relation}

The dispersion relation of the $2 \mathrm{P}-1 \mathrm{~B}$ analysis in shown in figure 6 by the green dotted line. The fastest growing 'traditional' two-dimensional HI has a growth rate higher than its three-dimensional confined counterpart CHI, at $\sigma_{r}=0.173$, which is achieved at a slightly larger wavenumber $k=1.52$, well within the experimentally range observed.

This mode is almost stationary, with a phase speed of $c=0.002$. This positive value is slightly surprising, as we are dealing with an originally leftward-propagating Holmboe mode. Further inspection reveals that this is an effect of the positive tilt angle $\theta=5^{\circ}$ of the duct. Solving with $\theta=0^{\circ}$ gives $\sigma_{r}=0.215, c=-0.045$. We found that a small positive angle decreases the growth rate and increases the phase speed to positive values. These effects are not thoroughly understood, but the behaviour of these linear, inherently twodimensional modes is not of central interest to our study. In contrast, the wavenumber and phase speed of the CHI predicted by the inherently three-dimensional 3P-2B analysis are not sensitive to the angle. For example, setting $\theta=0^{\circ}$ increases the growth rate slightly to $\sigma=0.10>0.0824$ but similar $k$ and $c$ are predicted.

\subsubsection{Eigenfunctions}

By analogy with the approach followed in $\S 6.1 .2$, the one-dimensional vorticity eigenfunction of the fastest growing mode was added to the one-dimensional base flow such that

$$
\omega_{y \dagger}^{*} \equiv \partial_{z} U+\alpha_{\dagger} \operatorname{Re}\left\{\hat{\omega}_{y \dagger}(z) \exp \left(i k x+\phi_{\dagger}\right)\right\}
$$

where the amplitude $\alpha_{\dagger}$ was determined in the same fashion as for the 3P-2B analysis, i.e. by matching the rms of the vorticity eigenfunction to the experimental perturbation in the midplane $y=0$ :

$$
\left\langle\left(\omega_{y \dagger}^{*}-\partial_{z} U\right)^{\mathrm{rms}}\right\rangle_{x, z}=\left\langle\left(\left\langle\omega_{y}\right\rangle-\partial_{z} U\right)^{\mathrm{rms}}(y=0)\right\rangle_{x, z}=0.58 .
$$

The other flow variables $\rho_{\dagger}^{*}, u_{\dagger}^{*}, w_{\dagger}^{*}$ were then obtained using $\alpha_{\dagger}$ similarly to $(6.3 a),(6.3 b)$, $(6.3 d)$ and are shown with $\omega_{y \dagger}^{*}$ in figure 10 .

From figure 10(a), it appears that the 'head' of the distinctive vorticity structure observed in the experimental CHW (figure 8(a)) and predicted by the CHI (figure 9(a)) is lacking. The vertical velocity pattern in figure 10(d) has a similar appearance to figures $8(\mathrm{~m})$ and $9(\mathrm{~m})$ but has a larger amplitude (approximately $60 \%$ larger, requiring different colour bar limits of \pm 0.1 instead of the previously used \pm 0.06$)$. The relative magnitude of the vertical velocity $w$ vs the spanwise vorticity $\omega_{y}$ (and hence the streamwise velocity $u$ ) in the $2 \mathrm{P}-1 \mathrm{~B}$ analysis is therefore different from the equivalent relative magnitude in the $3 \mathrm{P}-2 \mathrm{~B}$ analysis and poorly compares with the experimental observations. Moreover, this analysis is, by construction, incapable of reproducing the three-dimensional structure of the CHW discussed in 6.1.2, which we have shown depends on the spanwise coordinate $y$ and spanwise velocity $v$.

\subsection{Possible relevance to geophysical field observations}

The distinctive tilde-shape of the $\mathrm{CHW}$ and $\mathrm{CHI}$ is reminiscent of the field observations of Geyer et al. (2010) in the continuously forced stratified shear flow of the Connecticut River estuary. Through the combined use of acoustic backscattering and high frequency conductivity sensors around the sharp density interface (pycnocline), they observed a near-periodic signal of large sheared tilde-shaped regions of high density gradients with average wavelength around $10 \mathrm{~m}$ and height around $1 \mathrm{~m}$ (see their figures $2 \mathrm{~b}$ and $3 \mathrm{~b}$, 

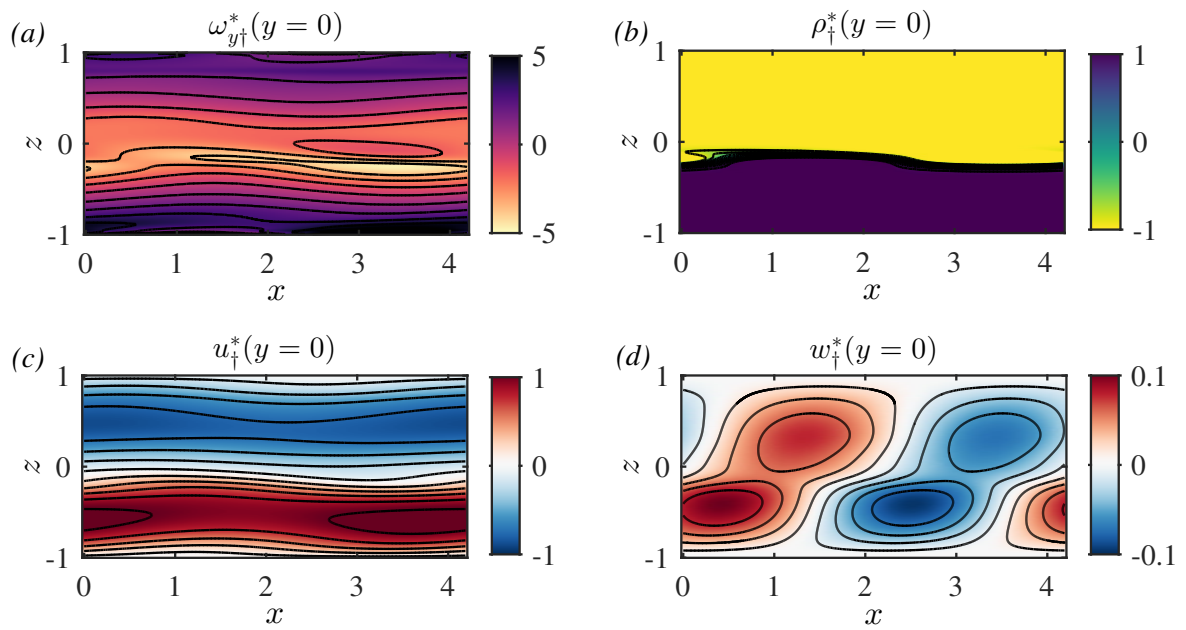

Figure 10. Fastest growing mode of the 2P-1B analysis (see text for definition of the variables). Note the higher colour bar limits of $\hat{w}$ when comparing with figures 8 and 9 . As in those previous figures, ten equally spaced black contours span the range of the colour bar for each panels.

where the $z$ axis has been stretched by factors of 12 and 6 , respectively). Those structures are oriented in the same direction as our vorticity structures (the mean shear has the same direction in both our work and their work) and we estimate their maximum angle at the inflection point around $5-6^{\circ}$, somewhat smaller than that of the $\mathrm{CHW}\left(10^{\circ}\right)$ and CHI $\left(8^{\circ}\right)$. They identified these regions as the braids connecting Kelvin-Helmholtz billows, and argued convincingly, in particular from the conductivity measurements, that these inclined 'braids' were the locations of the most vigorous turbulent motions, and that the large density gradients resulted from turbulent mixing within the braids, an inherently high-Re phenomenon which was unlikely to be observed experimentally or numerically. Mashayek \& Peltier $(2012 a, b)$ identified secondary instabilities localised in the braid regions connecting Kelvin-Helmholtz billows as a possible explanation for the observations of Geyer et al. (2010). Here we have identified a primary instability, the CHI, whose finite-amplitude manifestation is also consistent with these observations. Since the background profiles of the estuary flow are not known with sufficient accuracy to distinguish between flows susceptible to the Holmboe instability or flows susceptible to the Rayleigh instability, we cannot eliminate either of these explanations at this stage. However, unlike the simulated secondary braid instabilities of Mashayek \& Peltier $(2012 a, b)$, which occur as a transient event in an initial value problem, the observed CHWs occur in a flow in which the forcing is maintained in time as is the case in the estuary flow.

Preliminary experimental observations of flows in the stratified inclined duct in the intermittently turbulent and fully turbulent regimes revealed a variety of smaller-scale, shorter-lived vorticity structures whose appearance resembles that of the CHW. In particular, two-dimensional $(y=0)$ particle image velocimetry measurements at high temporal resolution in the intermittent regime $\left(\theta=4^{\circ}, R e=940\right.$ - not reported here) strongly suggest that those small-scale structures appear to come from the cascade break-down of larger-scale structures akin to the CHW through successive stretching and splitting.

We, therefore, argue that it is at least possible that the angled structures we observed experimentally $(\mathrm{CHW})$ and predicted theoretically (CHI), through their local coherent 
intensification of spanwise vorticity due to the spanwise confinement, also have the potential to leave a 'signature' at higher $R e$, even when incoherent, turbulent small scale motions have been triggered. In particular, in the spirit of the dynamical systems approach discussed in the introduction, we conjecture that the CHW might be sufficiently robust, even at substantially higher $R e$, to constitute an alternative nucleation site for secondary instabilities and hence turbulent break down, distinct from the strained braidlike region that occurs between finite amplitude Kelvin-Helmholtz billows proposed by Corcos \& Sherman (1976) and invoked by Geyer et al. (2010).

\section{Conclusions}

\subsection{Summary}

We have investigated the structure and origin of a 'confined Holmboe wave' (CHW), a finite-amplitude, nonlinear wave at the sharp density interface of a stratified shear flow.

The stratified inclined duct experiment (figure 1) sets up an exchange flow in an inclined square duct connecting two reservoirs containing fluids of different densities. The flow is steadily forced by gravity for hundreds of advective time units, and can maintain relatively high levels of dissipation, as measured by the buoyancy Reynolds number $R e_{b}$ (see (2.1)). By setting the duct tilt angle $\theta$ and the density difference $\Delta \rho$ between the two reservoirs connected by the duct (and hence the Reynolds number Re, see (3.4)), different flow states are observed. For $20 \lesssim R e \sin \theta \lesssim 50$, a coherent wave state features robust, propagating symmetric or asymmetric Holmboe waves (HWs), confined within the square cross-section of the duct. In order to characterise the structure of CHWs, we focused on the simpler case of upward-pointing, asymmetric HWs found in a flow in which $\theta=5^{\circ}$ and $R e=440$.

We employed a novel time-resolved, near-instantaneous measurement technique of the three-component velocity field $(u, v, w)$ (stereo particle image velocimetry) and density field $\rho$ (planar laser induced fluorescence) simultaneously over a three-dimensional volume (see figures 2, 3 and supplementary movies 1,2). This allowed us to reveal the spatiotemporal behaviour of this $\mathrm{CHW}$ (figures $4(\mathrm{a})$ and (b)) by quantifying its range of wavelengths $\lambda \in[3.5,6.0]$, or wavenumbers $k \in[1.05,1.80]$ (non-dimensionalised by half the duct height) and phase speeds $c \in[-0,18,0.05]$ (non-dimensionalised by the layer peak velocity). We first focused on the three-dimensional structure of its spanwise vorticity, where the wave field periodically reinforces and weakens the mean shear to form a pattern of inclined, distorted, prolate spheroids with a wide 'body' and a narrower 'head' (figure 3). Using phase averaging we extracted a typical, robust representation of the CHW and characterised its salient features (figures 4(c) and (d)).

To understand the origin (i.e. the underlying physical mechanisms that lead to the observed behaviour) of this apparently previously unreported structure, we undertook a linear stability analysis. We studied three-dimensional velocity $\left(u^{\prime}, v^{\prime}, w^{\prime}\right)$ and density $\rho^{\prime}$ perturbations having two-dimensional, cross-sectional eigenfunctions of $y, z$ and a normal streamwise mode in $x: \psi^{\prime} \equiv \hat{\psi}(y, z) \exp (i k x+\sigma t)$. Those perturbations were analysed on the two-dimensional ( $y, z$, i.e. $x$ and $t$-averaged) experimental mean flow (figure 5 ) and were confined with the appropriate boundary conditions in the square duct. This computation revealed the existence of a 'confined Holmboe instability' (CHI) with wavenumber band $k \in[0.8,2.2]$, phase speed band $c \in[-0.35,-0.08]$ and fastest growing mode $k=1.32, c=-0.21$, consistent with the properties of the CHW (figure 6). By matching the volume-averaged root-mean-square ( $\mathrm{rms}$ ) of the spanwise vorticity eigenfunction to the experimentally observed value, we reconstructed the three-dimensional structure of 
the CHI to compare it with the CHW. The side-by-side comparison of the vorticity isosurfaces (figures 4(c),(d) and 7(a),(b) as well as supplementary movie 3) and slices of flow variables $\omega_{y}, \rho, u, v, w$ (figures 8 and 9) shows excellent agreement between the finite-amplitude wave and the predicted instability. This view revealed further details including the spanwise mid-duct localisation, the convexity of the density crests and troughs, the resulting localisation of the baroclinic production of vorticity, as well as the divergence/convergence of the flow around density crests, causing vortex stretching. Such localisation and stretching are inherent to the spanwise confinement and are believed to play a critical role in the dynamics of the $\mathrm{CHI} / \mathrm{CHW}$.

We also compared these results with those of the more commonly used stability analysis of two-dimensional perturbations $\left(u^{\prime}, w^{\prime}, \rho^{\prime}\right)$ with an associated one-dimensional eigenfunction in $z$ and a streamwise normal mode in $x$ of the form $\psi_{\dagger}^{\prime} \equiv \hat{\psi}_{\dagger}(z) \exp (i k x+$ $\sigma t)$ on the one-dimensional, mid-duct base flow. Despite the dispersion relation showing some agreement (figure 6), the two-dimensional predicted structure (figure 10) compares less favourably with the experimental results and fails, by construction, to capture any spanwise confinement and behaviour. Crucially, the spanwise velocity perturbation $v^{\prime}$ of the $\mathrm{CHI}$ is an odd function of the spanwise $y$ coordinate, possessing a different spanwise periodicity from the other perturbation variables. This key property cannot be captured by such purely one-dimensional eigenfunctions, or even by normal modes 'propagating at an angle' to the base flow.

Finally, the coherent structure associated with the CHW bears a passing resemblance to the distinctive angled structures observed in the Connecticut River Estuary by Geyer et al. (2010). While perhaps fortuitous, this resemblance, as well as preliminary experimental results in more turbulent flows not reported here, lead us to conjecture that the angled coherent structures of the $\mathrm{CHW}$ may retain at least a partial signature in higher-Re flows with lateral confinement and sharp density gradients.

\subsection{Future directions}

Our results raise a number of questions that may stimulate future research.

(i) How generic is the effect of confinement on shear flow instabilities? This paper focused on an asymmetric Holmboe wave found in a particular laboratory flow, and four additional experiments with similar parameters confirmed that the qualitative results outlined in this paper are robust. Asymmetric HWs might be more generic than symmetric HWs due to some inevitable degree of asymmetry present in environmental flows; however, they introduce another nondimensional parameter (the degree of asymmetry) to the problem. More work is needed to investigate the effect of confinement in the parameter space spanned by HWs. Varying the level of confinement by considering rectangular ducts or other geometries would add further complexity but may be worth pursuing. Moreover, little work has been done to study the effect of confinement on other instabilities, such as the so-called Kelvin-Helmholtz instability. As outlined in our review in $\S 2$, laboratory observations in confined geometries are often compared to stability analyses that ignore confinement, and numerical simulations usually impose periodic boundary conditions in the spanwise direction. The results presented here, as well as other preliminary results, suggest that the properties of three-dimensional confined waves may differ significantly from those predicted by such analyses.

(ii) How is the amplitude of the $\mathrm{CHW}$ determined and sustained in time? The quasisteady forcing provided by the stratified inclined duct experiment sustains the observed CHW at an approximately constant amplitude for hundreds of advective time units. We determined this amplitude by computing the volume-averaged rms of the phase- 
average vorticity perturbation, and used it to compare with the most unstable linear eigenfunction. We did not address the value of this amplitude, which is selected through inherently nonlinear processes. By examining the structure of the CHW, we suggested possible nonlinear vorticity dynamics mechanisms (which rely on confinement) to sustain this wave (i.e. baroclinicity and vortex stretching) but their details and quantitative impact fall outside the scope of this paper. The relatively well-defined parameter range for which the $\mathrm{CHW}$ is observed is also particularly interesting. The wave studied here at $\theta=5^{\circ}, R e=440$ would be replaced by intermittent turbulence at slightly higher angles $\theta \gtrsim 6^{\circ}$ and/or values of $R e \gtrsim 600$. This suggests that the amplitude of this wave results from a fine-tuning of energy input (through $\theta$ ) and dissipation (through $R e$ ), and that the dissipation that the CHW structure can generate through higher amplitudes is no longer enough beyond a certain threshold in the $\theta-R e$ plane, at which it must bifurcate to more dissipative, intermittently turbulent, structures (Meyer \& Linden 2014; Lefauve 2018).

(iii) Is the coherent structure of the CHW dynamically relevant to more turbulent flows? Does this structure generically emerge in miniature around the fine scale density gradients of high-Reynolds number and high-Schmidt number confined flows such as estuarine flows discussed above? Can the large-scale coherent intensification of vorticity catalyse the formation of nucleation sites for secondary instabilities and turbulent break down? These considerations are central to the dynamical systems modelling mentioned in $\S 1$, using a reduced set of exact coherent states (ECSs), i.e. exact (yet unstable) nonlinear solutions of the Navier-Stokes equations. Recently, Lucas et al. (2017) successfully converged two ECSs from direct numerical simulations of body-forced, horizontally-sheared stratified turbulence in a triply-periodic domain. These ECSs were found to be striking representations of the mean flow and could account for the organisation of the stratified turbulence into inclined shear layers. By constructing a bifurcation diagram they demonstrated that the ECSs originate from a sequence of instabilities, including the stratified linear instability of the base flow. Our results suggest that the experimental long-lived coherent structure of the CHW originates from the linear CHI, but it is still unknown whether it is the signature of a relatively robust ECS that would carry its dynamical significance into confined stratified turbulent flows at geophysically relevant scales. We believe that this question warrants further investigation.

\section{Acknowledgements}

We acknowledge the support of EPSRC under the Programme Grant EP/K034529/1 'Mathematical Underpinnings of Stratified Turbulence' (MUST). We thank John Taylor, Rich Kerswell and the rest of the 'MUST team' for valuable discussions, as well as Lutz Lesshafft for suggesting the 3P-2B stability analysis. We are deeply indebted to the technicians of the G. K. Batchelor laboratory, David Page-Croft, Paul Mitton, Colin Hitch, John Milton and Andrew Denson for their craftsmanship.

\section{Appendix A. Derivation of the 3P-2B eigenvalue problem}

We start by combining the divergence of the linearised momentum equation $(5.1 b)$ and the linearised continuity equation $(5.1 a)$ to obtain a diagnostic equation for the pressure perturbation:

$$
-\nabla^{2} p^{\prime}=2\left(\partial_{y} U \partial_{x} v^{\prime}+\partial_{z} U \partial_{x} w^{\prime}\right)+R i\left(\cos \theta \partial_{z} \rho^{\prime}-\sin \theta \partial_{x} \rho^{\prime}\right) .
$$

We proceed to use (A 1) in combination with the Laplacian of the linearised momentum equation, in order to eliminate $p^{\prime}$. This reduces the dimensionality of our system by 
increasing its order. The resulting linear system (A 2) fully describes the time evolution of the perturbations using prognostic equations (A $2 b)$-(A $2 d$ ) for two velocity variables $v^{\prime}$ and $w^{\prime}$ and density $\rho^{\prime}$. The streamwise velocity perturbation $u^{\prime}$ is recovered independently by continuity (A $2 a)$ :

$$
\begin{aligned}
\partial_{x} u^{\prime}= & -\partial_{y} v^{\prime}-\partial_{z} w^{\prime} \\
\left\{\left(\partial_{t}+U \partial_{x}\right) \nabla^{2}+\left(\partial_{z z} U-\right.\right. & \left.\left.\partial_{y y} U\right) \partial_{x}+2 \partial_{z} U \partial_{x z}-R e^{-1} \nabla^{4}\right\} v^{\prime} \\
= & 2\left(\partial_{y z} U \partial_{x}+\partial_{z} U \partial_{x y}\right) w^{\prime} \\
& +R i\left(\cos \theta \partial_{y z}-\sin \theta \partial_{x y}\right) \rho^{\prime} \\
\left\{\left(\partial_{t}+U \partial_{x}\right) \nabla^{2}+\left(\partial_{y y} U-\right.\right. & \left.\left.\partial_{z z} U\right) \partial_{x}+2 \partial_{y} U \partial_{x y}-R e^{-1} \nabla^{4}\right\} w^{\prime} \\
= & 2\left(\partial_{y z} U \partial_{x}+\partial_{y} U \partial_{x z}\right) v^{\prime} \\
& +R i\left\{-\cos \theta\left(\partial_{x x}+\partial_{y y}\right)-\sin \theta \partial_{x z}\right\} \rho^{\prime} \\
\left\{\partial_{t}+U \partial_{x}-(R e S c)^{-1} \nabla^{2}\right\} \rho^{\prime}= & -\partial_{z} R w^{\prime} .
\end{aligned}
$$

We now transform the differential operators in $x$ and $t$ using our ansatz $(5.3)\left(\partial_{x^{n}}=\right.$ $\left.(i k)^{n}, \partial_{t}=\sigma\right)$, reducing (A $\left.2 b\right)-(\mathrm{A} 2 d)$ to the eigenvalue problem (5.4) and (A $2 a$ ) to (5.6).

\section{REFERENCES}

Armi, L. 1986 The hydraulics of two flowing layers with different densities. Journal of Fluid Mechanics 163, 27-58.

Baines, Peter G. \& Mitsudera, Humio 1994 On the mechanism of shear flow instabilities. Journal of Fluid Mechanics 276, 327-342.

Bartello, P. \& Tobias, S. M. 2013 Sensitivity of stratified turbulence to the buoyancy Reynolds number. Journal of Fluid Mechanics 725, 1-22.

Browand, F. K. \& WinANT, C. D. 1973 Laboratory observations of shear-layer instability in a stratified fluid. Boundary-Layer Meteorology 5 (1-2), 67-77.

Carpenter, J. R., Lawrence, G. A. \& Smyth, W. D. 2007 Evolution and mixing of asymmetric Holmboe instabilities. Journal of Fluid Mechanics 582, 103-132.

Carpenter, J. R., Tedford, E. W., Heifetz, E. \& Lawrence, G. A. 2013 Instability in Stratified Shear Flow: Review of a Physical Interpretation Based on Interacting Waves. Applied Mechanics Reviews 64 (6), 060801.

Carpenter, J. R., Tedford, E. W., Rahmani, M. \& Lawrence, G. A. 2010 Holmboe wave fields in simulation and experiment. Journal of Fluid Mechanics 648, 205-223.

Caulfield, C. P. 1994 Multiple linear instability of layered stratified shear flow. Journal of Fluid Mechanics 258, 255-285.

Caulfield, C. P., Peltier, W. R., Yoshida, Shizuo \& Ohtani, Morimasa 1995 An experimental investigation of the instability of a shear flow with multilayered density stratification. Physics of Fluids 7 (12), 3028.

Corcos, G. M. \& Sherman, F. S. 1976 Vorticity concentration and the dynamics of unstable free shear layers. Journal of Fluid Mechanics 73 (02), 241-264.

DrazIN, P. G. 1974 On a model of instability of a slowly-varying flow. Quarterly Journal of Mechanics and Applied Mathematics 27 (1), 69-86.

FARmer, D. \& ARMi, L. 1999 Stratified flow over topography: the role of small-scale entrainment and mixing. Proceedings of the Royal Society: Mathematical, Physical and Engineering Sciences 455, 3221-3258.

Geyer, W. R., Lavery, A. C., Scully, M. E. \& Trowbridge, J. H. 2010 Mixing by shear instability at high Reynolds number. Geophysical Research Letters 37 (22).

GiBson, C. H. 1980 Fossil temperature, salinity, and vorticity turbulence in the ocean. In Marine Turbulence (ed. J. Nihoul), pp. 221-257. Elsevier, Amsterdam.

Gibson, C. H. 1999 Fossil turbulence revisited. Journal of Marine Systems 21 (1-4), 147-167. 
Gibson, J. F., Halcrow, J. \& Cvitanović, P. 2008 Visualizing the geometry of state space in plane Couette flow. Journal of Fluid Mechanics 611, 107-130.

Haigh, S. P. \& Lawrence, G. A. 1999 Symmetric and nonsymmetric Holmboe instabilities in an inviscid flow. Physics of Fluids 11 (6), 1459-1468.

van Haren, H., Gostiaux, L., Morozov, E. \& Tarakanov, R. 2014 Extremely long KelvinHelmholtz billow trains in the Romanche Fracture Zone. Geophysical Research Letters 41 (23), 8445-8451.

HAZEL, P. 1972 Numerical studies of the stability of inviscid stratified shear flows. Journal of Fluid Mechanics 51 (01), 39-61.

Helmholtz, H. 1878 Über discontinuierliche Flüssigkeits-Bewegungen [On the discontinuous movements of fluids]. Monatsberichte der Königlichen Preussische Akademie der Wissenschaften zu Berlin 23, 215-228.

HogG, A. McC. \& Ivey, G. N. 2003 The Kelvin-Helmholtz to Holmboe instability transition in stratified exchange flows. Journal of Fluid Mechanics 477, 357-375.

Holmboe, J. 1962 On the behavior of symmetric waves in stratified shear layers. Geophys. Publ. 24, 67-113.

Ivey, G.N., Winters, K.B. \& Koseff, J.R. 2008 Density Stratification, Turbulence, but How Much Mixing? Annual Review of Fluid Mechanics 40 (1), 169-184.

Lawrence, G. A., Browand, F. K. \& Redekopp, L. G. 1991 The stability of a sheared density interface. Physics of Fluids A: Fluid Dynamics 3 (10), 2360-2370.

Lefauve, A. 2018 Stratified shear flows in an inclined duct. PhD thesis, University of Cambridge.

Lucas, D. \& CAulfield, C. P. 2017 Irreversible mixing by unstable periodic orbits in buoyancy dominated stratified turbulence. Journal of Fluid Mechanics 832, R1.

Lucas, D., Caulfield, C. P. \& Kerswell, R. R. 2017 Layer formation in horizontally forced stratified turbulence: connecting exact coherent structures to linear instabilities. Journal of Fluid Mechanics 832, 409-437.

LucAs, D. \& Kerswell, R. R. 2015 Recurrent flow analysis in spatiotemporally chaotic 2dimensional Kolmogorov flow. Physics of Fluids 27 (4), 045106.

Macagno, E. O. \& Rouse, H. 1961 Interfacial mixing in stratified flow. Journal of the Engineering Mechanics Division. Proceeding of the American Society of Civil Engineers 87 (EM5), 55-81.

Mahrt, L. 2014 Stably Stratified Atmospheric Boundary Layers. Annual Review of Fluid Mechanics 46 (1), 23-45.

Mashayek, A. \& Peltier, W. R. 2012a The 'zoo' of secondary instabilities precursory to stratified shear flow transition. Part 1 Shear aligned convection, pairing, and braid instabilities. Journal of Fluid Mechanics 708, 5-44.

Mashayek, A. \& Peltier, W. R. $2012 b$ The 'zoo' of secondary instabilities precursory to stratified shear flow transition. Part 2 The influence of stratification. Journal of Fluid Mechanics 708, 45-70.

Maxworthy, T. \& Browand, F. K. 1975 Experiments in rotating and stratified flows: oceanographic application. Annual Review of Fluid Mechanics 7, 273-305.

Meyer, C. R. \& Linden, P. F. 2014 Stratified shear flow: experiments in an inclined duct. Journal of Fluid Mechanics 753, 242-253.

Nishida, S. \& Yoshida, S. 1990 Influence of the density and velocity profiles on calculated instability characteristics in an inviscid two-layer shear flow. J. Hydrosci. Hydraul. Engng 7.

Olsthoorn, J. \& DAlziel, S. B. 2017 Three-dimensional visualization of the interaction of a vortex ring with a stratified interface. Journal of Fluid Mechanics 820, 549-579.

Partridge, J. L., Lefauve, A. \& Dalziel, S. B. 2018 A new method for near-instantaneous volumetric measurements of velocity and density fields. Submitted to Experiments in Fluids

Portwood, G. D., de Bruyn Kops, S. M., Taylor, J. R., Salehipour, H. \& Caulfield, C. P. 2016 Robust identification of dynamically distinct regions in stratified turbulence. Journal of Fluid Mechanics 807, R2.

Rayleigh, J. W. S. 1879 On the Stability, or Instability, of certain Fluid Motions. Proceedings of the London Mathematical Society s1-11 (1), 57-72. 
REYNOLDS, O. 1883 An experimental investigation of the circumstances which determine whether the motion of water shall be direct or sinuous, and of the law of resistance in parallel channels. Philosophical Transactions of the Royal Society of London 174, 935-982.

Salehipour, H., Caulfield, C. P. \& Peltier, W. R. 2016 Turbulent mixing due to the Holmboe wave instability at high Reynolds number. Journal of Fluid Mechanics 803, 591-621.

Smyth, W. D., Carpenter, J. R. \& Lawrence, G. A. 2007 Mixing in Symmetric Holmboe Waves. Journal of Physical Oceanography 37 (6), 1566-1583.

Smyth, W. D., Klaassen, G. P. \& Peltier, W. R. 1988 Finite amplitude holmboe waves. Geophysical \& Astrophysical Fluid Dynamics 43 (2), 181-222.

Smyth, W. D. \& Peltier, W. R. 1990 Three-dimensional primary instabilities of a stratified, dissipative, parallel flow. Geophysical \&S Astrophysical Fluid Dynamics 52 (4), 249-261.

Sмyтh, W. D. \& Peltier, W. R. 1991 Instability and transition in finite-amplitude KelvinHelmholtz and Holmboe waves. Journal of Fluid Mechanics 228, 387-415.

Smyth, W. D. \& Winters, K. B. 2003 Turbulence and mixing in Holmboe waves. Journal of Physical Oceanography 33 (4), 694-711.

Squire, H. B. 1933 On the stability of three-dimensional disturbances of viscous flow between parallel walls. Proceedings of the Royal Society A: Mathematical, Physical and Engineering Sciences 142, 621.

TAYlOR, G. I. 1931 Effect of Variation in Density on the Stability of Superposed Streams of Fluid. Proceedings of the Royal Society A: Mathematical, Physical and Engineering Sciences 132 (820), 499.

Tedford, E. W., Pieters, R. \& Lawrence, G. A. 2009 Symmetric Holmboe instabilities in a laboratory exchange flow. Journal of Fluid Mechanics 636, 137.

Thomson, W. 1871 Hydrokinetic solutions and observations. The London, Edinburgh, and Dublin Philosophical Magazine and Journal of Science 42 (281), 362-377.

Thorpe, S. A. 1968 A method of producing a shear flow in a stratified fluid. Journal of Fluid Mechanics 32 (04), 693-704.

Thorpe, S. A. 1971 Experiments on the instability of stratified shear flows: miscible fluids. Journal of Fluid Mechanics 46 (02), 299-319.

Woods, J. D. 1968 Wave-induced shear instability in the summer thermocline. Journal of Fluid Mechanics 32 (04), 791-800.

YIH, C. 1955 Stability of two-dimensional parallel flows for three-dimensional disturbances. Quarterly of Applied Mathematics 12 (4), 434-435.

Zhu, D. Z. \& Lawrence, Gregory A. 2001 Holmboe's instability in exchange flows. Journal of Fluid Mechanics 429, 391-409. 